\title{
The Influence of Periodic Forcing on the Time Dependence of Western Boundary Currents: Phase Locking, Chaos, and Mechanisms of Low-Frequency Variability*
}

\author{
ANDREW E. KISS \\ School of Physical, Environmental and Mathematical Sciences, University of New South Wales Canberra, Australian \\ Defence Force Academy, Canberra, Australian Capital Territory, and ARC Centre of Excellence for \\ Climate System Science, University of New South Wales, Sydney, New South Wales, Australia \\ LEELA M. FRANKCOMBE \\ Climate Change Research Centre, and ARC Centre of Excellence for Climate System Science, University of \\ New South Wales, Sydney, New South Wales, Australia
}

(Manuscript received 7 June 2015, in final form 24 November 2015)

\begin{abstract}
In this study an idealized gyre is put into a temporally periodic state by a steady wind stress curl forcing, and its nonlinear response to variable forcing is investigated by a detailed parameter survey varying the timemean component of the wind and the amplitude and frequency of a periodic component. Periodic wind variations exceeding $\sim 0.5 \%$ profoundly affect the western boundary current (WBC) time dependence, yielding regime diagrams with intricately interleaved regions of phase locking, quasiperiodicity, and chaos. In phase-locked states, the WBC period is locked to a rational multiple of the forcing period and can be shifted far outside its natural range. Quasiperiodic states can exhibit long intervals of near-synchrony interrupted periodically by brief slips out of phase with the forcing. Hysteresis and a period-doubling route to chaos are also found. The nonlinear WBC response can include variability at long time scales that are absent from both the forcing and the steadily driven current; this is a new mechanism for the generation of low-frequency WBC variability. These behaviors and their parameter dependence resemble the Devil's staircase found in the "circle map" model of a periodically forced nonlinear oscillator, but with differences attributable to higherdimensional dynamics. These nonlinear effects occur with forcing amplitudes in the observed range of the annual wind stress curl cycle and therefore should be considered when inferring the cause of observed WBC time scales. These results suggest that studies omitting either forcing variation or nonlinearity provide an unrealistically narrow view of the possible origins of time dependence in WBCs.
\end{abstract}

\section{Introduction}

Western boundary currents (WBCs) are regions of large heat transport and atmosphere-ocean heat fluxes, with very high variability on time scales ranging from seasonal to multidecadal, making them potentially

\footnotetext{
* Supplemental information related to this paper is available at the Journals Online website: http://dx.doi.org/10.1175/JPO-D-150113.s1.

Corresponding author address: Andrew Kiss, School of Physical, Environmental and Mathematical Sciences, UNSW Canberra, Australian Defence Force Academy, P.O. Box 7916, Canberra BC ACT 2610, Australia.

E-mail: a.kiss@adfa.edu.au
}

important in climate variability (Kelly et al. 2010; Kwon et al. 2010; Frankignoul et al. 2011; Hu et al. 2015). Here we address the causes of temporal variability in winddriven gyres, in particular whether a time scale observed in a WBC can be attributed (even in principle) entirely to an intrinsic instability in the current or to variability in the forcing. Process studies of WBC variability have mostly investigated the nonlinear dynamics of gyres under steady wind forcing, or the linear dynamics of gyres under variable winds, with relatively few including both nonlinearity and variable forcing. We therefore provide here a comprehensive study of the response of an idealized nonlinear subtropical gyre to periodic wind forcing. This provides insight into the robustness to external variability of the intrinsic WBC modes found under steady forcing, and also to 
the complexity that nonlinearity brings to the WBC response to forcing variation.

\section{a. Observed variability of western boundary currents}

Many aspects of observed WBC variability have been attributed to internal instability and/or external forcing. The Florida Current has an annual cycle with transport variations of $\pm 5 \%$ due to local wind forcing, and decadal variation partly due to variable wind stress curl associated with the North Atlantic Oscillation (NAO; Baringer and Larsen 2001; DiNezio et al. 2009). The Bermuda Current has a marked annual cycle in barotropic transport primarily driven by the basinscale wind stress curl (Lee et al. 1996). Farther downstream, the Gulf Stream is dominated by interannual time scales (Kelly et al. 2010). Path and transport variations are correlated with the NAO, with a weak annual cycle in phase with the Florida Current (Molinari 2004; Joyce et al. 2000). Interannual meridional variability in the Gulf Stream path also resembles an internal gyre mode (Speich et al. 1995; Simonnet et al. 2003; Dijkstra and Ghil 2005). Decadal variability in the position, pathlength, and volume transport of the Kuroshio and its extension have been linked to the arrival of baroclinic Rossby waves forced by central Pacific wind stress curl anomalies (Qiu and Chen 2005; Qiu 2003), although idealized models also show decadal internal variability under steady winds (Pierini et al. 2009).

The North Brazil Current (NBC), East Australian Current (EAC), and Agulhas Current retroflections shed large anticyclonic eddies about 8-9, 3-4, and 4-6 times per year, respectively (Johns et al. 2003; Mata et al. 2006; Beal et al. 2011), probably because of internal instability (Nof 1996; Bowen et al. 2005; Mata et al. 2006; Weijer et al. 2013). The EAC has a seasonal cycle in its transport and variance (Ridgway and Godfrey 1997; Qiu and Chen 2004), interannual variability linked to Rossby waves from the Tasman Sea, and multidecadal variation linked to waves from farther east (Holbrook et al. 2011; Hill et al. 2008; Sloyan and O'Kane 2015). ${ }^{1}$ Agulhas ring formation seems to be triggered by eddies from upstream in the Mozambique Channel, which in turn appear to be caused by arrival of Rossby waves from the Indian Ocean (Schouten et al. 2002). This upstream variability is modulated on interannual time scales by the Indian Ocean dipole (Palastanga et al. 2006). The Somali Current and East Indian Coast Current reverse direction between summer

\footnotetext{
${ }^{1}$ Recent analyses (Bowen et al. 2005; Mata et al. 2006) contradict suggestions by Nilsson and Cresswell (1980) and Marchesiello and Middleton (2000) that Rossby waves cause EAC eddy shedding.
}

and winter, partly because of a very strong monsoonal cycle in wind stress curl (Schott and McCreary 2001).

\section{b. Intrinsic $W B C$ variability with steady forcing}

Mechanisms proposed to explain WBC variability can be roughly divided into a forced response to variable winds (discussed later) or instabilities intrinsic to the current. Nonlinear mechanisms of intrinsic WBC variability have been extensively investigated using dynamical systems theory in idealized models under steady wind forcing, typically by surveying the dependence of the gyre's spatial structure and temporal variability on the Reynolds number Re (controlled by the wind forcing strength and eddy viscosity). With increasing $\mathrm{Re}$, a series of bifurcations from symmetrical to asymmetrical steady flow (with multiple equilibria) and then to periodic and aperiodic time dependence has been found in equivalentbarotropic shallow water (Jiang et al. 1995; Speich et al. 1995), 1.5- and 2-layer quasigeostrophic (Dijkstra and Katsman 1997; Berloff and McWilliams 1999), and 2(Nauw and Dijkstra 2001) and 2.5-layer (Simonnet et al. 2003) shallow-water double-gyre models, with time scales of intrinsic variability ranging from intra-annual to decadal. These studies and many others (see reviews by Dijkstra 2000; Dijkstra and Ghil 2005) provide a detailed understanding of the origins of variability and their time scales under steady forcing for near-laminar flows with only a few undamped modes. In the realistically turbulent regime, additional modes of low-frequency variability also arise (Dewar 2003; Berloff et al. 2007b).

\section{c. Ocean response to variable forcing}

Although such dynamical process studies provide many important insights, they omit any variability induced by the forcing, for example by spatiotemporal variation in the wind stress curl (WSC). In the North Pacific, WSC has an approximately white frequency spectrum over time scales of days to several months (Chave et al. 1991; Large et al. 1991). Annual and semiannual signals are also present and are strongest in the northern Indian, Pacific, and Atlantic Oceans, accounting for less than $45 \%$ of the WSC variance globally (Trenberth et al. 1990; Risien and Chelton 2008). Pronounced peaks rise above the broadband background noise at the annual frequency and a few harmonics, for example, in the North Atlantic (Hong et al. 2000; DiNezio et al. 2009), the North Pacific (Large et al. 1991; $\mathrm{Fu}$ 2004), and (especially) the northern Indian Ocean (Al Saafani et al. 2007), and an annual cycle in the Sverdrup transport is predicted from observed WSC in all midlatitude gyres (Trenberth et al. 1989), albeit with significant interannual variation. The dataset of Risien and Chelton (2008) shows that the amplitude of the 
annual cycle in the WSC is within an order of magnitude of the mean across most of the ocean and far exceeds the mean in the northern Indian Ocean. Longer-time-scale WSC variation also affects WBCs, as discussed above.

The Sverdrup balance and WBC return flow adjust to variations in wind forcing via Rossby waves; under linear conditions, the adjustment time scale is therefore set by the Rossby wave propagation time across the basin (Anderson and Gill 1975), which is of order several days for barotropic waves and a decade for the firstmode baroclinic waves. Rapid or short-wavelength atmospheric forcing cannot excite Rossby waves and therefore produces a local response, while very slow forcing (much longer than the Rossby wave basincrossing time) drives a time-dependent Sverdrup response. At intermediate frequencies, Rossby wave propagation yields a wavelike spatiotemporal response that may involve resonance with basin modes (Willebrand et al. 1980). The barotropic response is strongly influenced by topography on time scales shorter than the baroclinic adjustment (Anderson and Corry 1985). Under strongly nonlinear conditions, advective and turbulent processes determine the WBC adjustment time scale (Dewar 2003) and thereby set the time scale of coupled oceanatmosphere modes whose existence depends on the presence of eddies (Kravtsov et al. 2006; Berloff et al. 2007a).

Many studies of the response to variable forcing have used linear models lacking any internal variability (e.g., Anderson and Corry 1985; Myers and Weaver 1996; Sakamoto and Yamagata 1996; Qiu 2003; Qiu and Chen 2004, 2005; Holbrook et al. 2011) or have focused on the response to the stochastic component of the forcing in nonlinear models, either with (e.g., Sura et al. 2001; Schmeits and Dijkstra 2001; Pierini 2010) or without (e.g., Sapsis and Dijkstra 2013) intrinsic variability due to the steady component of the wind. Although some studies of the stochastic forcing have also included a periodic component (e.g., Chhak et al. 2009; Dewar 2003; Pierini 2011; and others cited below), there has been relatively little attention paid to the influence of the periodic component of the wind on nonlinear ocean models that have their own intrinsic variability. We therefore focus here on a simple ocean model (typical of those used in bifurcation studies) that is nonlinear but laminar, forced by wind with both a steady component (giving a periodic intrinsic state) and a periodic component, as a first step toward understanding the response of more realistic models.

\section{d. Periodic WBC with periodic forcing: Arnold tongues}

The response of a nonlinear model to periodic forcing is best understood by considering the trajectory of the model state vector. Under steady wind forcing of sufficient amplitude to cause periodic flow, the state vector's trajectory will repeatedly trace out a closed loop in phase space with a natural period $T_{n}$. If a small periodic oscillation is added to the forcing, the trajectory will wind around on the surface of a torus (formed by sweeping the periodic loop around to close on itself in a third dimension), completing one toroidal loop (the "long way" around the torus) per forcing period $T_{w}$ and one poloidal loop (the "short way" around) per period $T_{s}$, which is the average period of the system's response and may differ from $T_{n}$ (Glazier and Libchaber 1988).

If the winding number $T_{w} / T_{s}$ is exactly rational (say $a / b$, with no common factors) the system is periodicthe trajectory repeats after $b$ toroidal loops and $a$ poloidal loops, and a slice through the torus at a fixed forcing phase (a stroboscopic Poincaré section) shows $b$ intersections. With irrational $T_{w} / T_{s}$ the system is quasiperiodic: the trajectory winds densely over the torus, never closing on itself, and the Poincaré section has an infinite number of intersections lying on a closed loop (cf. Figs. 6 and 7). In a linear system $T_{s}=T_{n}$ and quasiperiodic behavior is found at almost all $T_{w} / T_{n}$; however, nonlinearity allows $T_{s}$ to shift away from $T_{n}$, yielding phase-locked periodic states in which $T_{w} / T_{s}$ is locked to a rational ratio across a range of $T_{w} / T_{n}$.

This kind of phase locking is found in many different contexts, and its generic features have mostly been studied in the simple "circle map" system that models the sequence of points in the stroboscopic Poincare section as a mapping from one angle $\phi_{k}$ on the loop to the next $\left(\phi_{k+1}\right)$, considered modulo $2 \pi$ :

$$
\phi_{k+1}=\phi_{k}+2 \pi T_{w} / T_{n}+\epsilon \sin \phi_{k},
$$

where $\epsilon$ controls the nonlinearity (Glazier and Libchaber 1988; Pikovsky et al. 2001; Rosenblum and Pikovsky 2003). Here $T_{w} / T_{s}=\lim _{k \rightarrow \infty}\left[\left(\phi_{k}-\phi_{0}\right) / 2 \pi k\right]$, which equals $T_{w} / T_{n}$ when $\epsilon=0$. For small $\epsilon>0$ there is a narrow range about each rational $T_{w} / T_{n}$ in which the oscillator is phase locked to the forcing. At a given $\epsilon$ these locked regions (called Arnold tongues) are typically wider for $a / b$ with smaller denominators, ${ }^{2}$ and they also widen as $\epsilon$ increases, as shown in Fig. 1. The line marking $\epsilon=1$ shows that $T_{w} / T_{s}$ repeatedly locks to rational values as $T_{w} / T_{n}$ increases, in a so-called "Devil's staircase." For $\epsilon \leq 1$ only locked and quasiperiodic states exist. For $\epsilon>1$ Eq. (1) is nonmonotonic and the dependence on parameters becomes increasingly intricate: tongues

\footnotetext{
${ }^{2}$ Tongue widths are actually ordered according to their level in the Farey tree.
} 


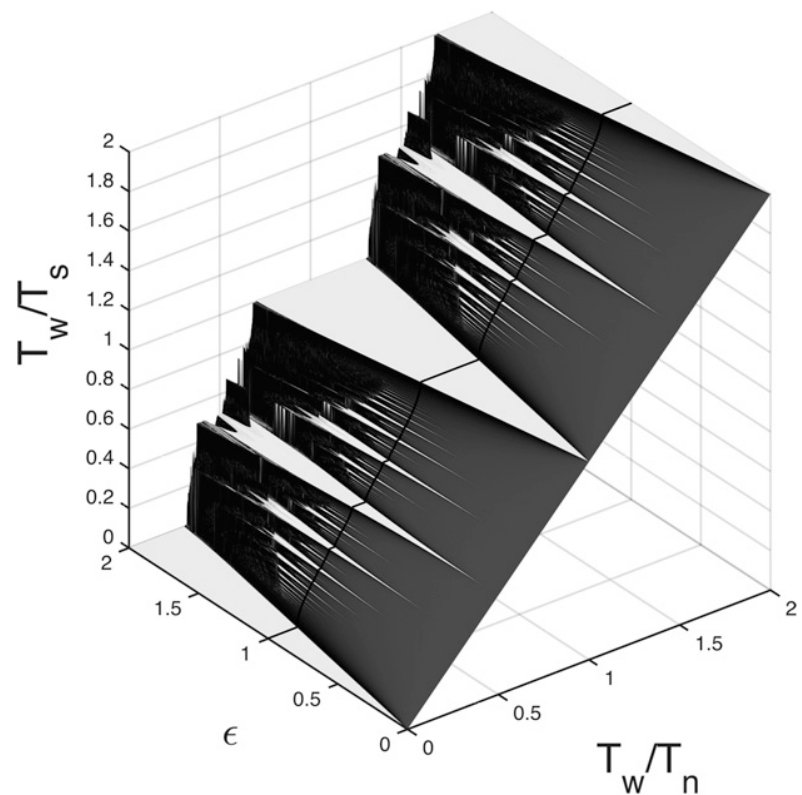

FIG. 1. Behavior of the circle map [Eq. (1)] as a function of $T_{w} / T_{n}$ and $\epsilon$. Phase-locked regions (Arnold tongues) are horizontal and appear light gray; they have rational $T_{w} / T_{s}$.

can overlap (i.e., hysteresis can occur, with different locking depending on the initial condition $\phi_{0}$ ), and only locked and chaotic states exist (Pikovsky et al. 2001). Although we use the circle map to provide a conceptual context for our experiments, only the behavior at small $\epsilon$ is generic, with the large- $\epsilon$ dynamics being model dependent.

A few previous studies have investigated the response of idealized ocean gyres to variable forcing in the light of circle-map theory. Shimokawa and Matsuura (2010) fixed the mean forcing at a value that gave periodic eddy shedding. When a periodic forcing was added (with $T_{w} / T_{n}$ fixed at 0.408), the gyre's time dependence could be quasiperiodic, phase locked $\left(T_{w} / T_{n}=1 / 2\right)$, or irregularly switch between bursts of eddy shedding and relative stability, depending on the amplitude of the forcing variability. Sakamoto (2006) used a very strong wind forcing variation (from zero to twice the mean) with $T_{w}$ fixed at 1 year. A parameter survey of forcing strength (over which $T_{n}$ varied by about $20 \%$ ) showed $T_{s}$ was locked over some intervals to a rational multiple of the forcing period, as expected from the Devil's staircase. Evidence was also found for phase locking between two modes of internal variability under steady, but stronger, forcing. Sushama et al. (2007) fixed an annual $10 \%$ wind variation and found a few cases of locking consistent with the Devil's staircase as the mean wind was varied.

Our study extends those of Shimokawa and Matsuura (2010) and Sakamoto (2006) by surveying a range of mean forcing strengths and forcing periods $T_{w}$, in addition to surveying a range of variability amplitudes. The relevant natural period $T_{n}$ is poorly known in the oceans and is a function of parameters in the model, so it is important to investigate a range of ratios $T_{w} / T_{n}$; we do this by varying $T_{w}$, which holds the characteristics of the WBC fixed and also allows us to explore a much greater range of frequency ratios than Sakamoto (2006). We also provide a detailed investigation of behavior under weakly varying winds, in contrast to Sakamoto (2006) and Shimokawa and Matsuura (2010), whose forcing variation was in most cases so large that it transiently crossed one or more of the stability boundaries that apply under steady forcing. This study builds on earlier work by Kiss and Ménesguen (2004) and Kiss (2007), but we provide here a much more comprehensive survey of parameter dependence. Our focus is on a very simple model of the wind-driven ocean circulation; a companion paper using a more realistic model is in preparation.

In section 2 we describe the idealized model. Section 3 gives results for steady forcing, the synchronization regimes under periodic forcing, and examples of the different types of behavior found with variable forcing. Section 4 contains a discussion and summary.

\section{The model}

We use the rigid-lid barotropic quasigeostrophic vorticity equation to model a single Northern Hemisphere midlatitude gyre driven by a time-dependent, spatially uniform wind stress curl in a circular basin with flat bathymetry. The simplicity of this model minimizes computational expense, allowing detailed multiparameter surveys to be undertaken. It is mathematically identical to the "sliced cylinder" laboratory model, which has been well characterized by numerical and laboratory studies of Pedlosky and Greenspan (1967), Beardsley $(1969,1975)$, Becker and Page (1990), Griffiths and Kiss (1999), Kiss (2002), and others. Anderson and Corry (1985), Willebrand et al. (1980), and Dewar (2003) show that the response of the ocean is barotropic at forcing periods less than about a year, so our model results are most relevant to the response of the ocean to annual and shorter forcing time scales. The horizontal flow is governed by the nondimensional vorticity equation

$$
\delta_{I}^{2}\left[\frac{\partial \zeta}{\partial t}+J(\psi, \zeta)\right]+\frac{1}{D} \frac{\partial \psi}{\partial x}=W-\frac{\delta_{S}}{D} \zeta+\delta_{M}^{3} \nabla^{2} \zeta
$$

with lateral boundary conditions of no throughflow ${ }^{3}$ and no slip,

\footnotetext{
${ }^{3}$ The boundary condition of McWilliams (1977) reduces to this form in the rigid-lid limit used here.
} 


$$
\psi=0, \quad \frac{\partial \psi}{\partial r}=0 \quad \text { on } \quad r=0.5,
$$

where $J$ is the Jacobian; $\psi$ is the streamfunction for the horizontal velocity; $\zeta=\nabla^{2} \psi$ is the relative vorticity; and $r, x$, and $y$ are the radial, eastward, and northward coordinates relative to the basin center. The anticyclonic wind forcing

$$
W=\left[-1+A \sin \left(f_{w} t\right)\right] / D
$$

includes a temporally periodic component of relative amplitude $A$ and frequency $f_{w}=2 \pi / T_{w}$, where $T_{w}$ is the period.

We include $D=1-\gamma y$, where $\gamma=s L / H=0.784$, for consistency with Kiss and Ménesguen (2004) and Kiss (2007), who model the apparatus of Griffiths and Kiss (1999) in which $\beta$ is simulated by a bottom slope $s$ in a tank of central depth $H$ and diameter $L$, producing depth variations that alter the stretching terms. Our results are nearly identical to those with $D=1$ (e.g., Kiss 2002) because $D$ cancels out in the Sverdrup and Stommel balances that govern the flow in much of the domain (Kiss 2003, 2004).

As in Kiss and Ménesguen (2004), in Eqs. (2) and (4), lengths have been scaled by the basin diameter $L$ (so $-0.5<y<0.5$ ), $\psi$ is scaled by the Sverdrup streamfunction $\psi_{\mathrm{Sv}}=W^{*} L / \beta_{0}$, and time $t$ by the advective time scale $L^{2} / \psi_{\mathrm{Sv}}$, where $W^{*}$ is the dimensional vorticity tendency due to Ekman pumping by the wind and $f_{0}$ and $\beta_{0}$ are the dimensional Coriolis parameter and its northward gradient at the basin's center. The flow under steady forcing is governed by the dimensionless inertial WBC width $\delta_{I}=\sqrt{\psi_{S v} / \beta_{0} L^{3}}$ (controlled by the mean wind forcing strength) and the dimensionless Stommel and Munk WBC widths $\delta_{S}=\left(\beta_{0} L H\right)^{-1} \sqrt{A_{V} f_{0} / 2}$ and $\delta_{M}=L^{-1}\left(A_{H} / \beta_{0}\right)^{1 / 3}$, where $A_{V}$ and $A_{H}$ are the vertical and horizontal eddy viscosities. Griffiths and Kiss (1999) and Kiss (2007) used different scaling, in which the dimensionless parameters are an unconventional Rossby number ${ }^{4}$ Ro $=4(L /$ $H)^{2}\left(\delta_{M} / \delta_{S}\right)^{3} \delta_{I}^{2}$ [this corrects an error in Kiss (2002) and Kiss and Ménesguen (2004), whose reported values for $\delta_{I}$ were too large by a factor of $\sqrt{2}$ ], the Ekman number $E=4(L / H)^{4} \delta_{M}^{6} / \delta_{S}^{2}$ (Ekman layers occur on both the top and bottom boundaries in their tank, giving an extra factor of 2), and the bottom slope $s=2(L / H) \delta_{M}^{3} / \delta_{S}^{2}$.

\footnotetext{
${ }^{4}$ This definition of Ro cannot be equated with the ratio of relative to planetary vorticity. We investigate $\mathrm{Ro}<0.11$, but relative vorticity significantly alters the WBC potential vorticity structure (see Fig. 4a of Kiss 2002).
}

We present results for fixed values $\delta_{S}=1.0102 \times$ $10^{-2}$ and $\delta_{M}=8.6660 \times 10^{-3}$, so the WBC occupies a small fraction of the basin width (as in the oceans), and both Ekman and lateral friction are significant in its vorticity balance. This corresponds to $E=6.273 \times$ $10^{-5}, L / H=7.84$, and $s=0.1$ as used in Kiss (2007). With these parameters fixed, Ro $=155.22 \delta_{I}^{2}$. The value of $\delta_{I}$ reflects the mean wind stress curl, which sets the natural time dependence of the WBC. We choose fixed values of $\delta_{I}$ between $1.7 \times 10^{-2}$ and $2.6 \times 10^{-2}$ and survey the response to different wind variability (i.e., different $A$ and $f_{w}$ ). The width $\delta_{I}$ exceeds $\delta_{S}$ and $\delta_{M}$, so inertia strongly modifies the Stommel and Munk WBC balances (Kiss 2000). Table 1 expresses these parameters in terms of dimensional scales, showing that we are in a comparable region of parameter space to previous dynamical systems studies in a small ocean basin (e.g., Speich et al. 1995; Nadiga and Luce 2001). As in Ierley and Sheremet (1995), the wind stress and curl are larger than observed (e.g., Risien and Chelton 2008; DiNezio et al. 2009) in order to obtain realistic WBC transport in a small basin whose zonal/meridional aspect ratio is smaller than in the oceans; the indicative maximum stress is also larger for a given curl because of its linear profile.

The model used to solve Eq. (2) is derived from a code by Michael Page (Monash University) and uses a polar grid (512 azimuthal $\times 161$ radial points, amply resolving $\delta_{I}, \delta_{S}$, and $\delta_{M}$ ), with second-order conservative finite differences in space and time (details are given in Kiss 2000). The natural period $T_{n}$ was very well resolved with roughly 1000 time steps per $T_{n}$ for most runs, and convergence tests show that the model period is within $0.2 \%$ of the true (continuous time) value (Kiss 2007). The forcing period $T_{w}=2 \pi / f_{w}$ was always chosen to be an integer number of time steps, so the discretized form of Eq. (2) was exactly periodic. We focus on the long-term temporal behavior well after spinup from initial conditions (usually from rest) and after many forcing cycles.

\section{Results}

\section{a. Bifurcation diagram under steady forcing}

Under steady forcing $(A=0)$ the flow regimes are a function of Ro, as well as the other parameters $(E, L / H$, and $s$ ) that we hold fixed (Griffiths and Kiss 1999; Kiss 2000). For weak forcing (small Ro) the flow is steady and the western boundary current is north-south symmetric. It separates to form an asymmetric jet as Ro increases (Kiss 2002), until at a critical value $\mathrm{Ro}=\mathrm{Ro}_{c 1}$ the jet becomes unstable and begins to oscillate periodically, which develops into periodic shedding of cyclonic eddies 
TABLE 1. Examples of dimensional ocean scales matching our dimensionless parameters. All cases have $\delta_{S}=1.0102 \times 10^{-2}, \delta_{M}=$ $8.6660 \times 10^{-3}, f_{0}=7.29 \times 10^{-5} \mathrm{~s}^{-1}, \beta_{0}=2 \times 10^{-11} \mathrm{~s}^{-1} \mathrm{~m}^{-1}$, and density $\rho=1025 \mathrm{~kg} \mathrm{~m}^{-3}$. Wind stress varying linearly from $-\tau_{\max }$ to $\tau_{\mathrm{max}}$ across the basin will have the required uniform curl. Blanks have the same value as to the left.

\begin{tabular}{|c|c|c|c|c|c|c|}
\hline Basin diameter $L(\mathrm{~km})$ & 1000 & & & 2000 & & \\
\hline Basin depth $H_{0}(\mathrm{~m})$ & 4000 & & & 1000 & & \\
\hline "Rossby number" Ro (see footnote 4 on the previous page) & 0.048 & 0.06 & 0.08 & 0.048 & 0.06 & 0.08 \\
\hline Dimensionless inertial scale $\delta_{I}$ & 0.01759 & 0.01966 & 0.02270 & 0.01759 & 0.01966 & 0.02270 \\
\hline Stommel WBC scale, $L \delta_{S}(\mathrm{~km})$ & 10.10 & & & 20.20 & & \\
\hline Munk WBC scale, $L \delta_{M}(\mathrm{~km})$ & 8.67 & & & 17.33 & & \\
\hline Inertial WBC scale, $L \delta_{I}(\mathrm{~km})$ & 17.59 & 19.66 & 22.70 & 35.17 & 39.32 & 45.40 \\
\hline Ekman spindown time scale, $1 /\left(\beta_{0} L \delta_{S}\right)$ (days) & 57.3 & & & 28.6 & & \\
\hline Horizontal friction, $A_{H}=\beta_{0} L^{3} \delta_{M}^{3}\left(\mathrm{~m}^{2} \mathrm{~s}^{-1}\right)$ & 13.0 & & & 104.1 & & \\
\hline Sverdrup velocity, $U=\beta_{0} L^{2} \delta_{I}^{2}\left(\mathrm{~mm} \mathrm{~s}^{-1}\right)$ & 6.18 & 7.73 & 10.31 & 24.74 & 30.92 & 41.23 \\
\hline Sverdrup transport, $L H_{0} U(\mathrm{~Sv})$ & 24.7 & 30.9 & 41.2 & 49.5 & 61.8 & 82.5 \\
\hline Sverdrup advective time scale, $L / U$ (days) & 1871.4 & 1497.1 & 1122.8 & 935.7 & 748.5 & 561.4 \\
\hline Natural eddy-shedding period, $T_{n}$ (days) & 166.21 & 96.62 & 65.88 & 83.16 & 48.31 & 32.94 \\
\hline Shortest Rossby basin mode period, $T_{0,1}$ (days) & 34.98 & & & 17.49 & & \\
\hline Wind stress curl, $-H_{0} \beta_{0} \rho U\left(\times 10^{-7} \mathrm{~Pa} \mathrm{~m}^{-1}\right)$ & -5.07 & -6.34 & -8.45 & -5.07 & -6.34 & -8.45 \\
\hline Wind stress maximum, $\tau_{\max }=L H_{0} \beta_{0} \rho U / 2(\mathrm{~Pa})$ & 0.25 & 0.32 & 0.42 & 0.51 & 0.63 & 0.85 \\
\hline
\end{tabular}

at larger Ro (see Fig. 2). More complex temporal behavior emerges at even larger Ro (see below).

Our primary diagnostic is the time series of basinintegrated kinetic energy

$$
K(t)=\frac{1}{2} \iint u^{2}+v^{2} d x d y,
$$

where $u$ and $v$ are the horizontal components of velocity. The kinetic energy density $(1 / 2)\left(u^{2}+v^{2}\right)$ is dominated by the WBC and separated jet. With $A=0$ the unseparated part of the WBC is nearly steady and the variation in $K$ is primarily due to the eddy-shedding process at the end of the jet. Figure 3 shows power spectra of $K$ as a function of Ro, which can be interpreted as a bifurcation diagram (Nadiga and Luce 2001). Horizontal white lines indicate the periods of linear, inviscid Rossby basin modes in the absence of mean flow, ${ }^{5}$ given by $T_{m n}=\left(8 \pi k_{m n}\right) /\left(L \beta_{0}\right)$ (dimensional), where $k_{m n}$ is the $n$th real zero of the $m$ th-order Bessel function of the first kind, for integers $m \geq 0$ and $n \geq 1$ (Pedlosky and Greenspan 1967).

The transition from steady to periodic flow occurs via a supercritical Hopf bifurcation at $\mathrm{Ro}=\mathrm{Ro}_{c 1}$ (between 0.047 and 0.048), that is, the least-damped mode is oscillatory and is decaying for $\mathrm{Ro}<\mathrm{Ro}_{c 1}$, but it is a sustained oscillation whose amplitude increases continuously from zero with increasing $\mathrm{Ro}>\mathrm{Ro}_{c 1}$ (see Fig. 5 in Kiss 2007). For $\mathrm{Ro}>\mathrm{Ro}_{c 1}$ the flow spins up to an

\footnotetext{
${ }^{5}$ The flow speed exceeds the Rossby wave phase speed only in the narrow WBC and jet, so it is not expected to significantly alter the frequency of the large-scale modes.
}

oscillatory state with a stable amplitude (blue circles in Fig. 3) that generally increases with Ro and whose period drops monotonically from about $3.802 T_{0,1}$ at $\mathrm{Ro}_{c 1}$ to $2.160 T_{0,1}$ at $\mathrm{Ro}=0.072$; only one period (and its harmonics) is present in this range. Physically, this oscillation is periodic shedding of cyclonic eddies from the first meander of the separated western boundary current jet (Fig. 2). Although the geometry differs, the dimensional eddy-shedding period $T_{n}$ (Table 1 ) is similar to the time scales of roughly $100,60-90$, and 40-45 days at which anticyclones are shed from the unseparated EAC, Agulhas, and NBC, respectively (Mata et al. 2006; Beal et al. 2011; Johns et al. 2003), via a mixed barotropic/baroclinic instability for the Agulhas and EAC (Weijer et al. 2013; Mata et al. 2006).

Several bifurcations occur between Ro $=0.072$ and $\mathrm{Ro}=0.0735$, which we collectively denote $\mathrm{Ro}_{c 2}$. From Ro $=0.0725$ to Ro $=0.0734$ there are two states, depending on initial conditions. State 1 is the continuation of the oscillation from $\mathrm{Ro}_{c 1}$. This becomes quasiperiodic at $\mathrm{Ro}=0.0725$, where an additional incommensurate period 3.170 $T_{0,1}$ appears [presumably because of a second Hopf bifurcation between Ro $=0.072$ and 0.0725 , as in van der Vaart et al. (2002)], and is then chaotic from $\mathrm{Ro}=0.0726$ to $\mathrm{Ro}=0.0734$, beyond which it disappears . The chaotic state is "bursty," lingering near the quasiperiodic state from $\mathrm{Ro}=0.0725$ but erratically diverging from it and returning (as in Pomeau-Manneville intermittency), so its broad spectral peaks align with those of the quasiperiodic state (Fig. 3b). The coexisting state 2 is periodic, with a different period of $2.690 T_{0,1}$ and a larger standard deviation (Fig. 3b), possibly arising from a saddle-node bifurcation between $\mathrm{Ro}=0.072$ and 


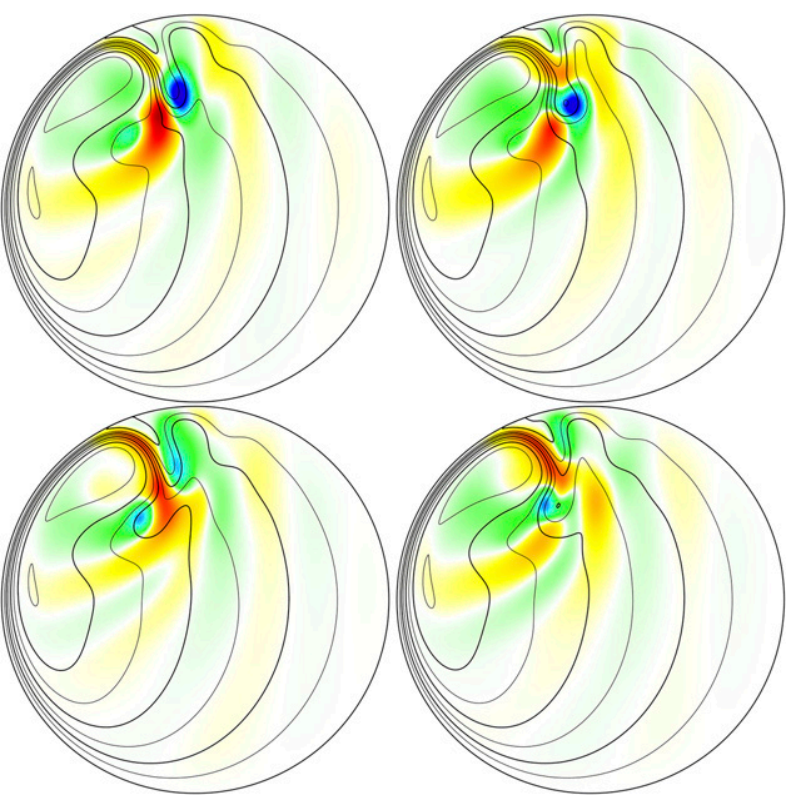

FIG. 2. One eddy-shedding cycle at Ro $=0.06\left(\delta_{I}=0.01966\right)$ under steady forcing (time progresses clockwise). The $\psi$ contours are overlaid on $\psi-\bar{\psi}$ (blue, green $<0<$ yellow, red), where $\bar{\psi}$ is the streamfunction for the unstable steady state at the same parameters (see Kiss 2007).

Ro $=0.0725$ (e.g., Nadiga and Luce 2001, their Fig. 15). Only state 2 is found for Ro $>0.0734$. This has a perioddoubling bifurcation at $\mathrm{Ro}_{c 3}$ between 0.088 and 0.09 , and for $\mathrm{Ro}>\mathrm{Ro}_{c 4}$ (between 0.098 and 0.099) the time dependence is chaotic, with power across a wide range of low frequencies in addition to broadened peaks aligned with the state 2 periods at smaller Ro. A complex EOF analysis (not shown) reveals that the spatial structure of the oscillation changes continuously with Ro, so the bifurcations in Fig. 3 primarily represent changes in the temporal (not spatial) behavior. In particular, across $\operatorname{Ro}_{c 2}$ there is a transition between two spatially similar modes of different period, similar to the two "gyre modes" seen in two-gyre models (van der Vaart et al. 2002).

Figure 3 shows no evidence of resonance or locking at basin mode periods $T_{m n}$; this is unsurprising, as Ekman friction gives a low quality factor $Q_{m n}=1 /\left(8 k_{m n} \delta_{S}\right) \leq$ 5.15 (Beardsley 1975). However, $T_{1,1}, T_{2,1}, T_{3,1}$, and $T_{4,1}$ seem to be related to the $T_{n}$ at which bifurcations occur (no $T_{m 2}$ modes are involved, presumably because they have a nodal circle at the radius where eddies are shed; Pedlosky and Greenspan 1967).

\section{b. Response of intrinsically periodic state to periodic forcing}

As a first step in studying the influence of variable external forcing on the internal variability, we apply wind forcing [Eq. (4)] that varies sinusoidally with period $T_{w}$ and relative amplitude $A$, with the strength of the time-mean winds controlled by Ro. Wind variation excites Rossby waves that propagate toward the western boundary [see Hovmöller diagrams in Kiss (2007)] where they cause periodic variation in the WBC strength that may alter the timing of eddy shedding. This is a periodically forced nonlinear oscillator, with the intrinsic period $T_{n}$ arising because of the instability of the jet. The nonlinearity of the coupling between the flow and the forcing is controlled by both Ro and $A$; thus, for a given Ro, $A$ will play a role akin to the nonlinearity parameter $\epsilon$ in the circle map [Eq. (1) and Fig. 1].

A major objective of this paper is to elucidate the conditions under which these forced variations of the WBC strength can alter or control the timing of eddy shedding in the separated jet, in particular to identify the boundaries between phase-locked, quasiperiodic, and chaotic regimes (see section 1d). The dimensionality of this system is far too high for a Floquet analysis to be practical (van der Vaart et al. 2002), so instead we performed over 4500 experiments, surveying a range of amplitudes $A$ of the forcing variability and ratios $T_{w} / T_{n}$ between the forcing and natural periods, at several values of the mean wind forcing strength (i.e., different Ro). We used the method of Kiss (2007) to search for exponentially converging locked states at every possible locking ratio in our finite-length $K(t)$ time series (a far more stringent test for locking than simply examining spectra). Unlocked states were categorized as quasiperiodic or chaotic by analyzing $K(t)$ Fourier spectra, delayspace embeddings, and Poincaré sections strobed at the forcing period. The results for different values of the mean forcing Ro are shown in Fig. 4, with details of individual cases shown in section 3d. Regime diagrams for Ro $<\mathrm{Ro}_{c 1}$ are not shown since they were all $n: 1$ locked.

At $\mathrm{Ro}=0.048$ (just above $\mathrm{Ro}_{c 1}$ ) we find that all the simulations are either phase locked or quasiperiodic (Fig. 4a). This low Ro yields a weakly nonlinear WBC jet oscillation of small amplitude (Fig. 3a). For small $A$, phase locking occurs only near $T_{w} / T_{n}=0.5$ (1:2 locking, i.e., the WBC oscillation repeats exactly once every two forcing cycles) and for a wider range of values near $T_{w} / T_{n}=1(1: 1$ locking), and $T_{w} / T_{n}=2$ at somewhat larger $A$ (2:1 locking). At the edge of the 1:1 tongue a relative wind variation $A$ of only $1 \%$ is sufficient to shift the jet period $T_{s}=T_{w}$ by over $30 \%$ from its natural value $T_{n}$, equivalent to a $25 \%$ increase in the steady component of the wind (Ro $>0.06$; Fig. 3). The width of the tongues increases with $A$, particularly the $1: 1$ tongue that grows to encompass the much narrower $1: 2$ tongue; with a $10 \%$ variation the jet period can be reduced to $T_{n} / 4$, a value unobtainable at any Ro under steady forcing. Eventually $n: 1$ locking 

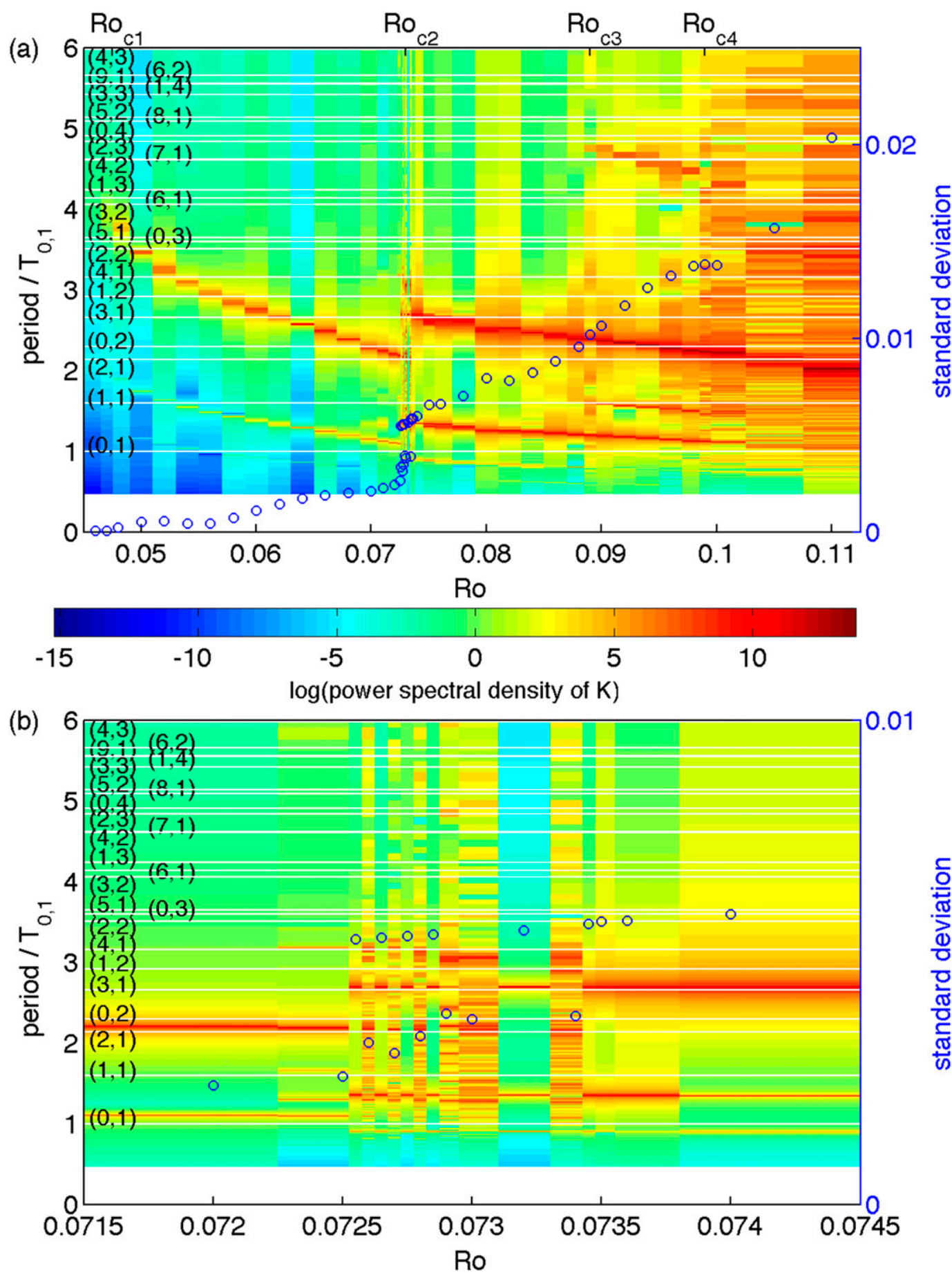

FIG. 3. (a),(b) Log of power spectral density of $K$ (colors) as a function of steady wind forcing strength Ro (bottom axis) and period in units of the shortest Rossby basin mode period $T_{0,1}$ (left axis). Standard deviation of $K$ (blue circles, right axis) is overlaid as a function of Ro; these also indicate the Ro value at which each spectrum is calculated. Horizontal lines indicate periods of linear inviscid Rossby basin modes [labeled by $(m, n)$ ] in the absence of mean flow. There are two states (state 1: quasiperiodic/chaotic, and state 2: periodic, with larger standard deviation) in the range Ro $=0.0725$ to 0.0734 , shown in more detail in (b); we depict this by interleaving the spectra and standard deviations of the two states by slightly offsetting state 2 to the right in this Ro range (so their true Ro values are given by the blue circle of state 1 to their left). 
$\mathrm{Ro}=0.048$

(a)

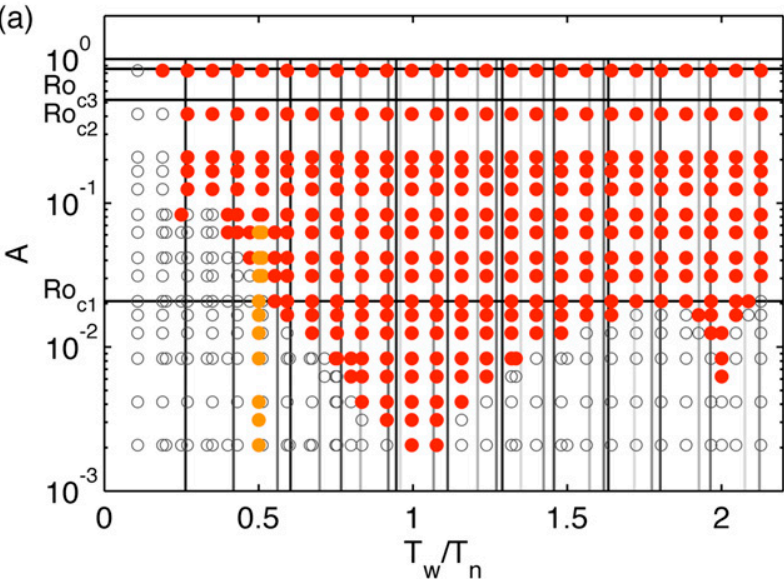

(c)

10

$\mathrm{Ro}=0.07$

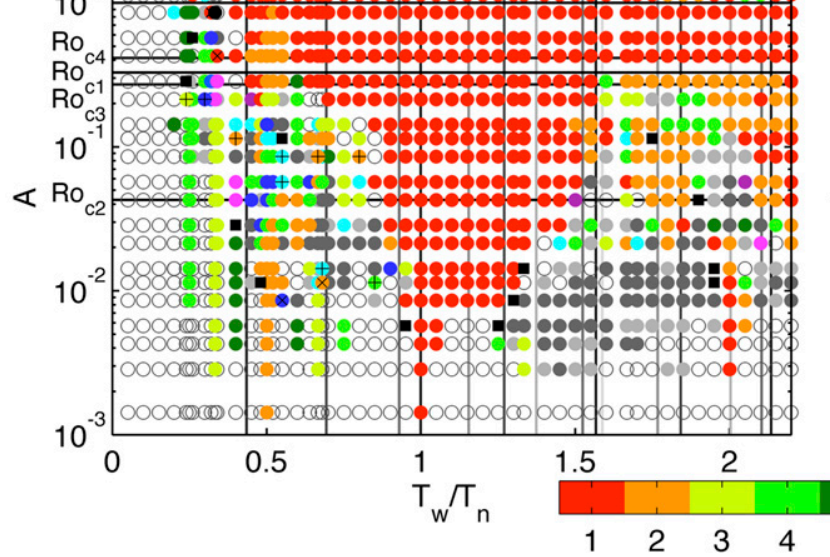

\section{(b)}

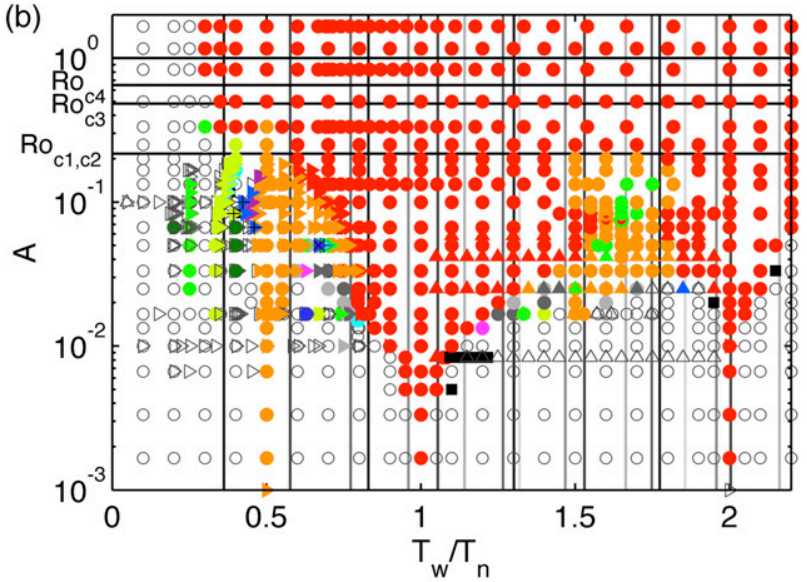

$\mathrm{Ro}=0.08$

(d)

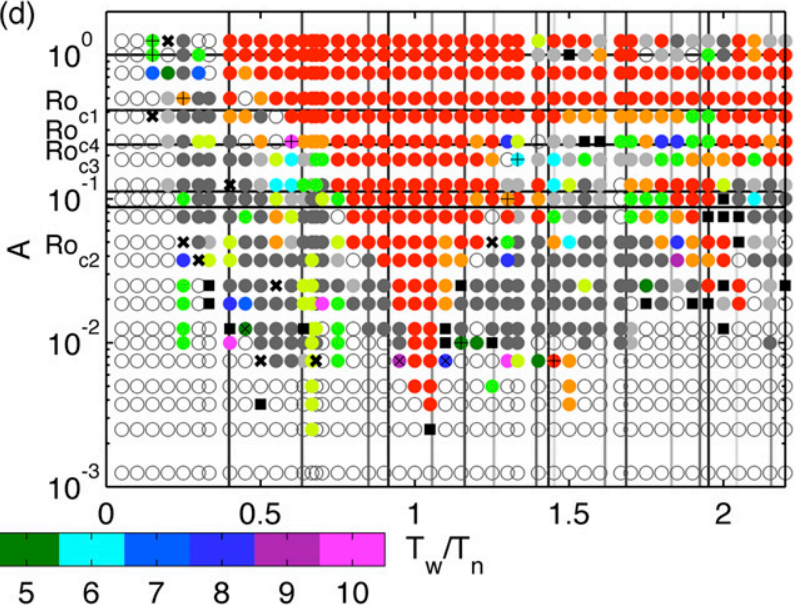

FIG. 4. Regime diagrams showing final state as a function of relative forcing amplitude $A$ and forcing period $T_{w}$ scaled by natural period $T_{n}$, for mean forcing Ro of (a) Ro $=0.048\left(\delta_{I}=0.01759, T_{n}=3.802 T_{0,1}\right)$, just above the critical value $\operatorname{Ro}_{c 1} ;(\mathrm{b}) \operatorname{Ro}=0.06\left(\delta_{I}=0.01966, T_{n}=\right.$ $\left.2.762 T_{0,1}\right)$; (c) Ro $=0.07\left(\delta_{I}=0.02124, T_{n}=2.297 T_{0,1}\right)$; and (d) $\operatorname{Ro}=0.08>\operatorname{Ro}_{c 2}\left(\delta_{I}=0.02270, T_{n}=2.511 T_{0,1}\right)$. Open symbols are quasiperiodic and black squares represent quasiperiodic cases with prominent phase slipping. Colored symbols are phase-locked states (the color scale indicates the denominator of $T_{w} / T_{s}$ in reduced form, that is, the number of forcing cycles between exact repetitions). Colored circles with a $+(\times)$ symbol are locked to $10+(20+)$ the color scale (e.g., a light blue circle represents $n: 6$ locking but a light blue circle with an overlaid + represents $10+6$, i.e., $n: 16$ locking), and large black $\times$ symbols represent locking with a denominator exceeding 30 . Cases where a different final state was found after starting from a nearby point (section 3c) are shown by a colored ring around the circle (the central color was obtained from rest). Solid gray symbols are chaotic (type 1 and 2 are light and dark gray, respectively), and triangles are runs from Kiss and Ménesguen (2004) and Kiss (2007). Vertical lines indicate Rossby basin mode periods $T_{m n}$ (shaded by $m$ ). The total wind forcing changes sign above $A=1$; the $A$ values at which the forcing crosses $\operatorname{Ro}_{c 1,2,3,4}$ in part of the forcing cycle are also marked.

covers the whole parameter space apart from a small region at small $T_{w} / T_{n}$. No locking of order higher than $1: 2$ is found, but this does not rule out the possibility since Fig. 1 suggests this would occur in extremely narrow tongues and thus be difficult to locate. In the unlocked quasiperiodic states, both the forcing and natural frequencies (and their cross harmonics) were present in the spectra of $K$.

More complex Arnold tongues become detectable as Ro increases further beyond $\mathrm{Ro}_{c 1}$ and the eddy-shedding limit cycle becomes more nonlinear. At Ro $=0.06$
(Fig. 4b) the regime diagram displays higher-order tongues, chaos, and phase-slipping quasiperiodicity (discussed below), while still retaining the prominent tongues near $T_{w} / T_{n}=0.5,1$, and 2 seen at Ro $=0.048$ (although the 1:1 tongue is now narrower and the 1:2 tongue is wider). Kiss and Ménesguen (2004) and Kiss (2007) presented similar (but much sparser) regime diagrams; their results are shown as triangles in Fig. 4b; some of their runs used a longer time step and their consistency with our results gives reassurance that the model's temporal resolution is adequate. 


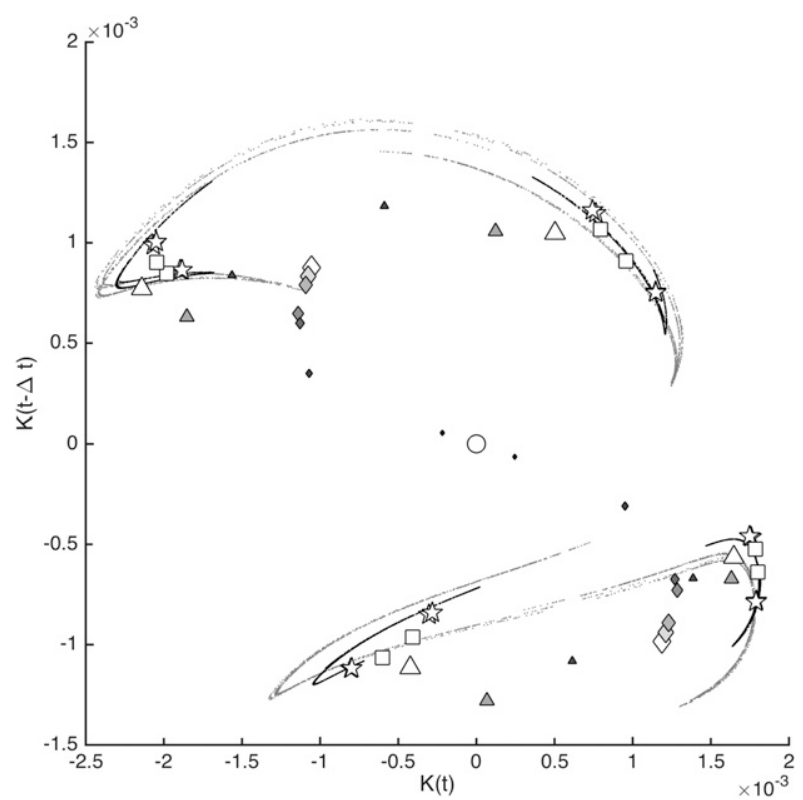

FIG. 5. Stroboscopic Poincaré sections for Ro $=0.06, A=0.05$, showing a period-doubling route to chaos with decreasing $T_{w} / T_{n}$. A 1:1 locked state (circle, $T_{w} / T_{n}=0.7701$ ) becomes unstable via a period-doubling bifurcation, from which a stable $n$ :2-locked state arises (diamond pairs, shown in increasing size and lightness for $\left.T_{w} / T_{n}=0.7664,0.7482,0.7299,0.7263,0.7190,0.7153,0.7117\right)$. This in turn period-doubles to $n: 4$ (triangles, shown in increasing size and lightness for $\left.T_{w} / T_{n}=0.7080,0.7007,0.6934\right)$, then $n: 8$ (squares, $\left.T_{w} / T_{n}=0.6898\right)$, and $n: 16\left(\operatorname{stars}, T_{w} / T_{n}=0.6861\right)$. Type 1 chaos with a strong $n: 4$ component appears at $T_{w} / T_{n}=0.6825$ (four curves of black dots) and fills more of delay space at $T_{w} / T_{n}=0.6788$ (gray dots), with a foliated structure. The mean has been subtracted, stroboscopic sampling is at each wind forcing peak, and delay $\Delta t=T_{w} / 4$ in all cases. The time step for these runs was 4 times longer than usual.

At $\mathrm{Ro}=0.06$ the structure of locked tongues resembles that found in the circle map, particularly when $T_{w} / T_{n}<1$. Quasiperiodicity prevails at small $A$, except for tongues at 1:2,1:1, and 2:1. Tongues at 2:5, 1:3, and $1: 4$ are also detected at higher $A$, as is a single case at $1: 5$ consistent with a tongue. Some higher-order tongues bend with increasing $A$, as is commonly seen in forced nonlinear oscillators. The 1:3 tongue bends so far that it no longer straddles the expected value $T_{w} / T_{n}=1 / 3$. The $1: 2$ tongue also shows asymmetry; this slight bending is not seen in the circle map, but its structure is not generic at finite $\epsilon$. The circle map regime structure (Fig. 1) repeats exactly with increasing $T_{w} / T_{n}$ and is symmetric about $T_{w} / T_{n}=0.5$, because Eq. (1) depends on $T_{w} / T_{n}$ modulo 1 and its nonlinear part is antisymmetric under a change of sign of $\phi_{k}$. Figure 4 shows that our system lacks these exact symmetries.

The 1:2 tongue in Fig. $4 \mathrm{~b}$ is forked, with one part extending to low $A$ (at $T_{w} / T_{n}=0.5$ ) as expected for an
Arnold tongue, but the other part abutting the low- $T_{w} /$ $T_{n}$ edge of the 1:1 tongue only down to about $A=0.03$. This second region arises from period doubling from the 1:1 tongue. A detailed transect at $A=0.05$ (see Fig. 5) shows a period-doubling route to chaos with decreasing $T_{w} / T_{n}$, going from $1: 1$ to $n: 2, n: 4, n: 8, n: 16$, and finally chaos with finely foliated and apparently fractal Poincaré sections. ${ }^{6}$ The spacing of the bifurcations is in quantitative agreement with the Feigenbaum constant to within the limited precision of the parameter survey. We expect period doubling explains other similar features at tongue edges, for example, the $n: 6$ region to the right of the 1:3 tongue in Fig. 4b, and other chaotic states near tongue edges.

We make a qualitative distinction between two categories of chaos. In type 1 chaos the points in the Poincaré section lie on or near a closed curve, as would be expected for chaos arising from a single forced oscillator. In type 2 chaos no such curve is found, and instead the points form a cloud in the Poincaré section, indicating that the trajectory no longer lies on a torus, and so higher-dimensional dynamics are important. In Fig. 4 types 1 and 2 are marked in light and dark gray, respectively, and examples are shown in Figs. 5 and 9.

For higher values of the mean forcing $(\mathrm{Ro}=0.07,0.08$ in Figs. 4c,d) the 1:1 tongue becomes increasingly narrow, that is, higher amplitudes of forcing variability are required to cause 1:1 locking at a significantly mismatched forcing frequency, indicating that the intrinsic oscillation is more robust. Multiple locked tongues are detectable (particularly at Ro $=0.07$ ), but chaos of both types is much more common and is induced by forcing variations of order $1 \%$ across a wide range of parameters. Type 2 chaos becomes increasingly prevalent, suggesting that the torus is more easily disrupted, perhaps because other modes of the flow are less strongly damped than at lower Ro. At Ro $=0.07$, the $1: 2$ tongue is indistinct for $A>0.02$ and period-doubled ratios of $n: 4, n: 8$, and $n: 16$ predominate; $1: 2$ reappears at $A>0.2$, possibly via period doubling from the $1: 1$ tongue. The structure at Ro $=0.08$ generally appears less ordered, perhaps because of stronger dependence on initial conditions (see section 3c), and the 1:2 tongue has almost disappeared. Figures $4 \mathrm{c}$ and $4 \mathrm{~d}$ nevertheless show a close resemblance, despite having crossed the bifurcation at $\mathrm{Ro}_{c 2}$, which increased $T_{n}$ (Fig. 3).

\footnotetext{
${ }^{6}$ Our $n: d$ notation simply indicates that repetition takes $d$ forcing cycles to repeat. The $m$ th period doubling splits the torus into $2^{m}$ overlapping sheets, increasing $d$ by the factor $2^{m}$ without altering the ratio of loops the long and short ways around the torus; the winding number is therefore unchanged (Glazier and Libchaber 1988).
} 
The distribution of period doubling and chaos along tongue edges in Figs. $4 \mathrm{~b}$ and $4 \mathrm{c}$ is not consistent with the routes to chaos in the circle map, in which chaos arises either abruptly from quasiperiodic states at $\epsilon=1$ or by period doubling in the interiors of locked tongues with $\epsilon>1$ (Glazier and Libchaber 1988). The relationship between $\epsilon$ and $A$ is probably nonlinear and also dependent on $T_{w} / T_{n}$, as tongue overlap (starting at $\epsilon=1$ ) seems to occur at larger $A$ for smaller $T_{w} / T_{n}$, but it is clear that chaos arises at lower $A$ than tongue overlap.

Type 1 chaotic states tend to occur close to tongues (or close to $T_{w} / T_{n}$ with small denominator), whereas type 2 tend to occur in regions where quasiperiodicity is found at smaller Ro. We speculate that type 2 chaotic states may be due to excitation of an otherwise-damped oscillatory mode by the variable forcing, giving three frequencies and a Ruelle-Takens-Newhouse transition to chaos (Newhouse et al. 1978). The presence of higherorder dynamics is evident from the type 2 Poincaré sections and is also consistent with the mismatch between our regime diagrams and those for the circle map: higher-dimensional maps in other systems (e.g., Aronson et al. 1986; Haucke and Ecke 1987) display some features of the circle map, but with important differences.

The Rossby basin modes (vertical lines in Fig. 4) have no significant influence on the regime structure. Horizontal lines in Fig. 4 indicate forcing variations strong enough to transiently cross the thresholds $\mathrm{Ro}_{c 1,2,3,4}$ identified under steady forcing (Fig. 3). These also appear to have no influence on the regime structure, probably because $T_{w} / T_{n}$ does not greatly exceed unity, so there is insufficient time for the WBC to adjust in the fraction of the cycle that crosses the threshold (such thresholds become relevant only with much larger $T_{w}$ and $A$; see section $3 \mathrm{e}$ ). It is important to note that chaos is induced by surprisingly weak forcing variation, far weaker than is required to transiently cross the $\mathrm{Ro}_{c 4}$ threshold (or enter the narrow $\mathrm{Ro}_{c 2}$ region) at which chaos appears under steady forcing, and at mean wind forcing also well below the threshold $\mathrm{Ro}_{c 4}$.

\section{c. Dependence on initial conditions}

The simulations in Fig. 4 were spun up from rest. However, the circle map suggests that neighboring locked tongues may "overlap" at large nonlinearity, so that either locked state can be obtained from suitable initial conditions in the overlap region. We found three cases where the final state depended on the initial condition, shown in Fig. 4b by different colored outlines on the circles near $T_{w} / T_{n}=1.6, A=0.08$. These were $1: 1$ locked when started from rest but had a different locking (either $n: 2$ or $n: 4$ ) when started from nearby spun-up runs with that locking (with the forcing phase adjusted to remain continuous). The presence of this hysteresis is evidence that locked tongues exist in this region, whose extension to small $A$ was presumably too narrow to detect.

Extensive testing of different initial conditions was not attempted; however, we expect overlap to be common near the edges of tongues at large $A$; if so, the tongue boundaries in Fig. 4 may not represent what would be found for spinup from a state other than rest. Such overlap may explain some of the apparent interleaving of tongues near their edges, since the tongue on which a solution lands (after spinup from rest) may differ at different parameters. However, as discussed above, some of the abutting locked regions are due to period doubling rather than tongue overlap, and in these cases dependence on initial conditions would not be expected.

\section{d. Examples of behavior under time-dependent forcing}

We now present examples of $K(t)$ diagnostics from a few cases of interest. Note that $K$ is not sensitive to the forcing-generated Rossby waves while they are in transit, but when they arrive at the western boundary they create significant spatially coherent variations in the kinetic energy density in the unseparated WBC, giving a strong signal in $K$ at the forcing period $T_{w}$. In contrast, the process of interest (eddy shedding) mainly involves relocation of regions of high kinetic energy density, and so appears as a relatively weak signal in the integral $K$ (a point measurement of vorticity in this region is much more sensitive to eddies; see Figs. 8, 10).

Figure 6 shows a case that locks at $T_{w}: T_{s}=1: 3$. The $K$ power spectrum (Fig. 6a) has peaks at the eddyshedding frequency $f_{s}=2 \pi / T_{s}=f_{w} / 3$ and at $2 f_{s}$ and $f_{w}$. Phase locking is evident in Figs. $6 \mathrm{~b}$ and $6 \mathrm{c}$, which show the Poincare section and kinetic energy anomaly over many forcing cycles, but is most convincingly demonstrated by the diagnostic of Kiss (2007) (not shown).

Figure 7 shows a typical quasiperiodic case. This has a line spectrum with large peaks at both the natural and forcing frequencies and numerous minor peaks at sums and differences of their harmonics (which also contribute power at much lower frequency). The absence of locking is evident in Fig. 7c, which shows the $K$ anomaly sweeps through a continuous range at any given phase of the forcing, reflecting the phase shift of the $K$ anomaly relative to the forcing in Fig. 7d. The intersections in the Poincaré section (Fig. 7b) progress in an orderly sequence around a closed loop, at a fairly constant pace that reflects a nearly uniform shifting of phase relative to the forcing. Movies of $K$ density anomaly in similar 
(a)

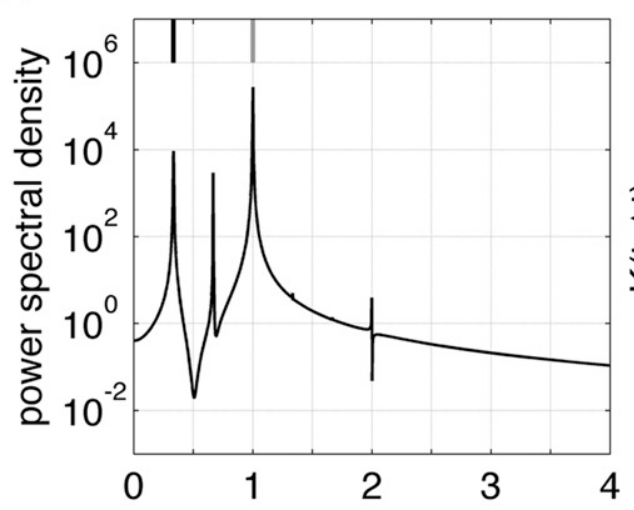

Frequency (cycles per forcing period) (b) Poincare section

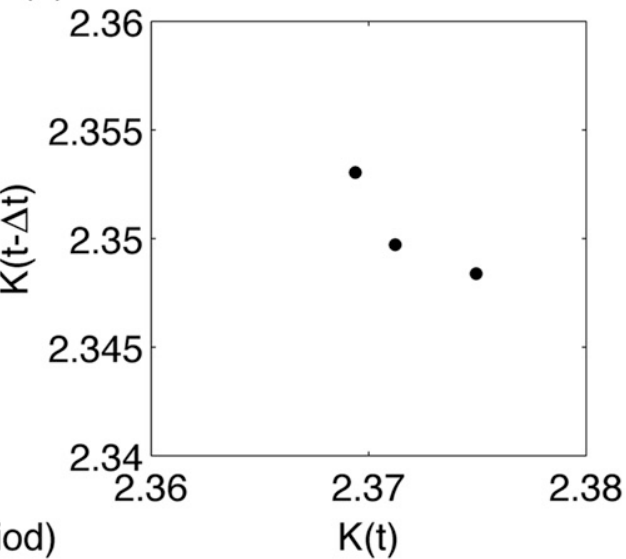

(c)

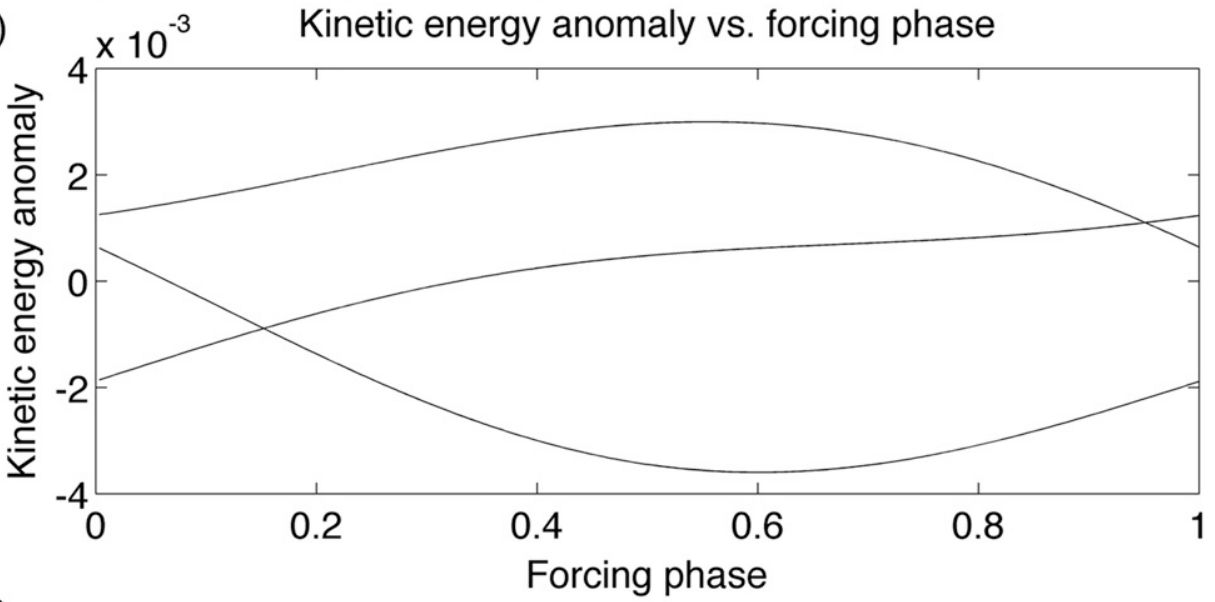

(d)

Kinetic energy and forcing

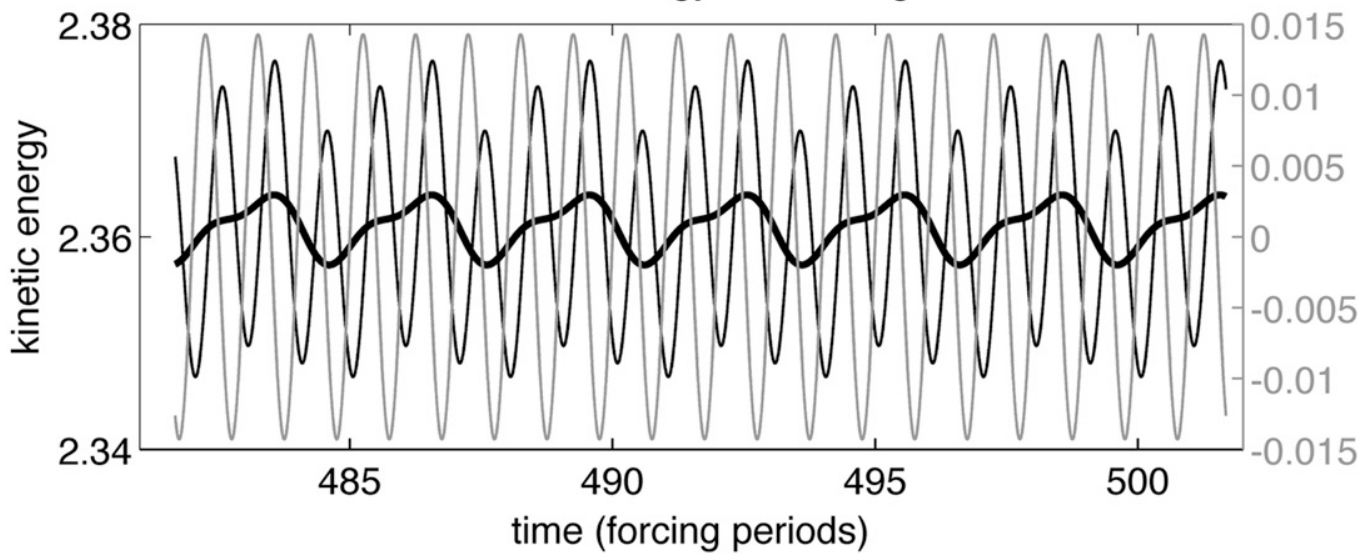

FIG. 6. The 1:3 locking for Ro $=0.07\left(\delta_{I}=0.02124\right), A=0.01429, T_{w} / T_{n}=0.33$. (a) Power spectrum of kinetic energy $K$ with the forcing (natural) frequency indicated by a gray (black) bar; (b) stroboscopic Poincaré section (sampled at the forcing period) of delay-space embedding of $K$, with delay $\Delta t=1.280 T_{0,1}$ between the axes with grayscale (from light to dark) representing time; (c) the anomaly of $K$ (relative to its average cycle over a forcing period) vs forcing phase (running from 0 to 1 ), with grayscale (from light to dark) representing time; and (d) time series of kinetic energy (thin black line; left axis), the anomaly of $K$ relative to its average cycle over a forcing period (thick black line; left axis, offset by mean $K$ ), and forcing relative to the mean (gray line; right axis). 
(a)

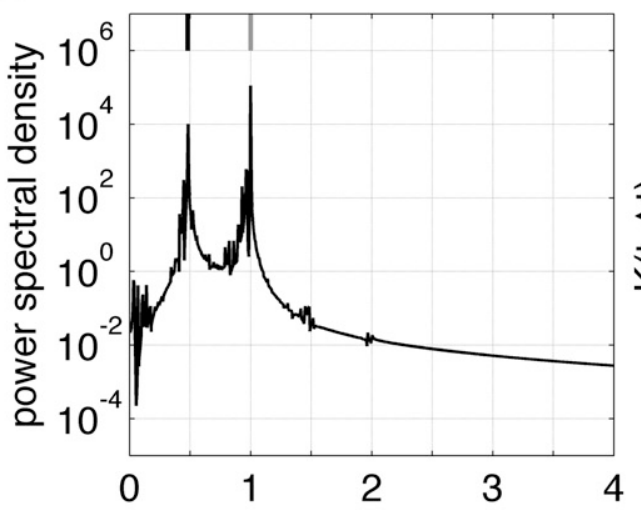

Frequency (cycles per forcing period) (b)

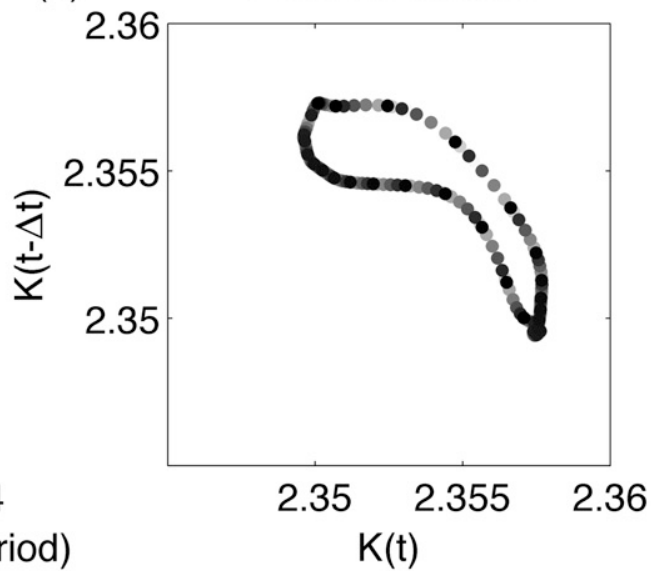

(c)

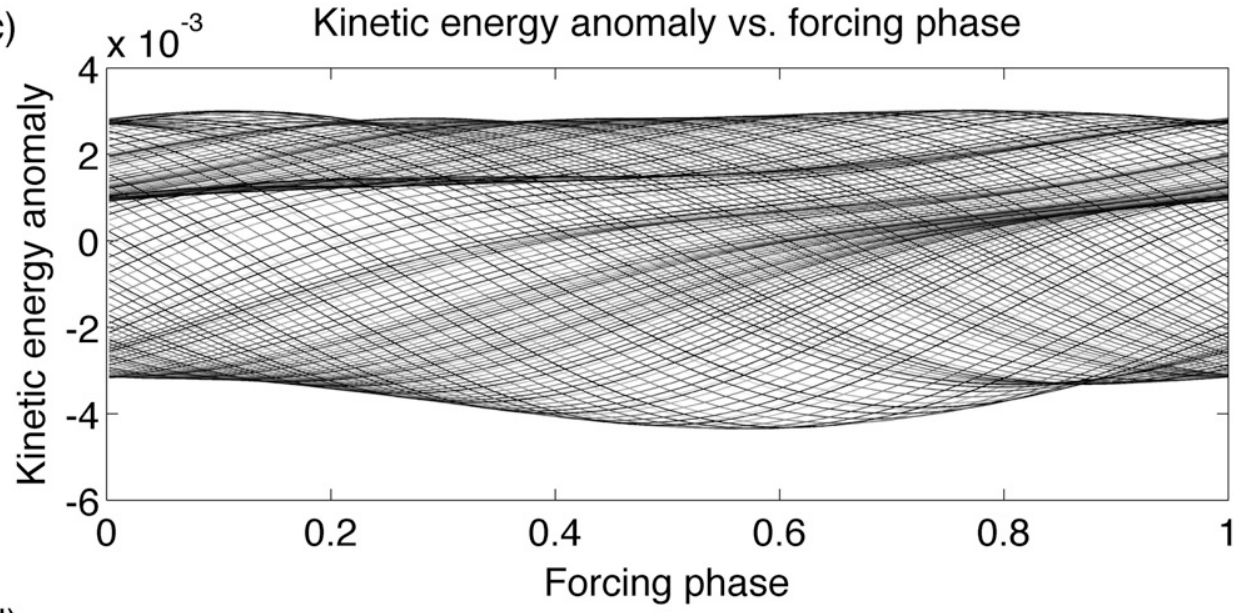

(d)

Kinetic energy and forcing

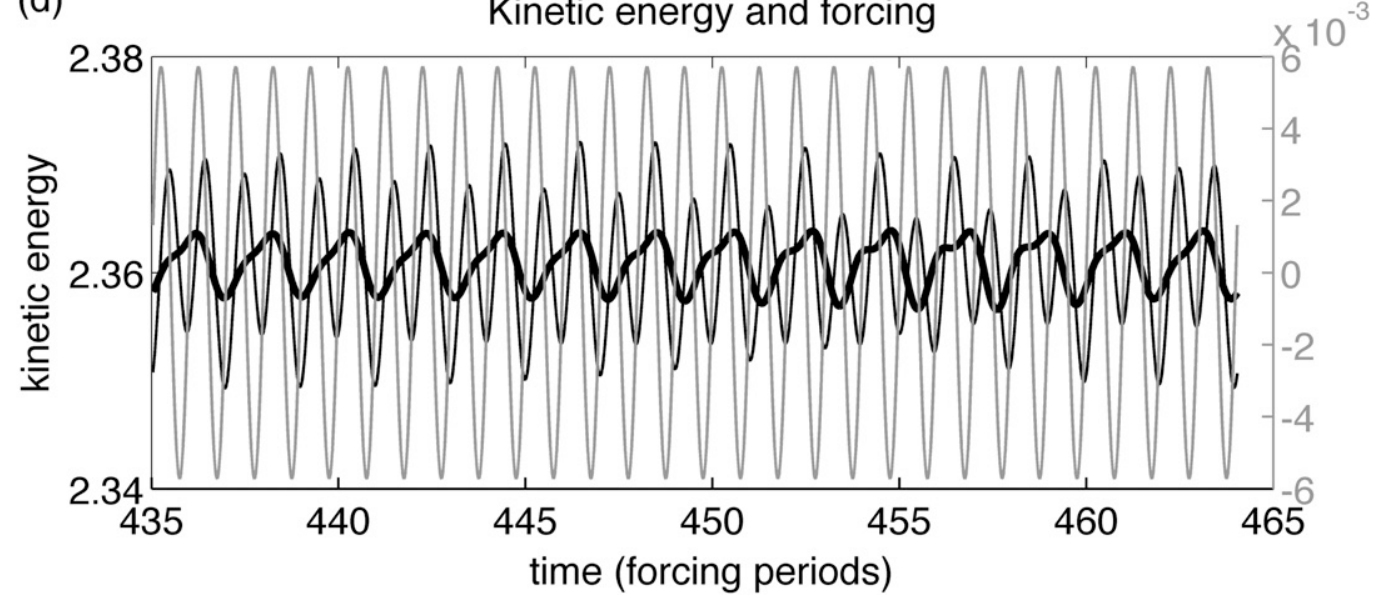

FIG. 7. As in Fig. 6, but for quasiperiodic behavior for Ro $=0.07\left(\delta_{I}=0.02124\right), A=5.714 \times 10^{-3}, T_{w} / T_{n}=0.48$.

quasiperiodic runs show variability at the forcing frequency in the WBC, but this does not extend far into the jet and does not alter eddy-shedding timing, which proceeds at the (slower) natural frequency, giving two independent fundamental frequencies in the spectrum.
Phase-slipping behavior is observed under quasiperiodic conditions close to locking, with intervals of nearlocking periodically interrupted by brief slips out of phase with the forcing. These slips appear in the spectrum as a peak at the difference frequency $\left|f_{w}-f_{s}\right|$, indicating a nonlinear modulation of the eddy shedding 


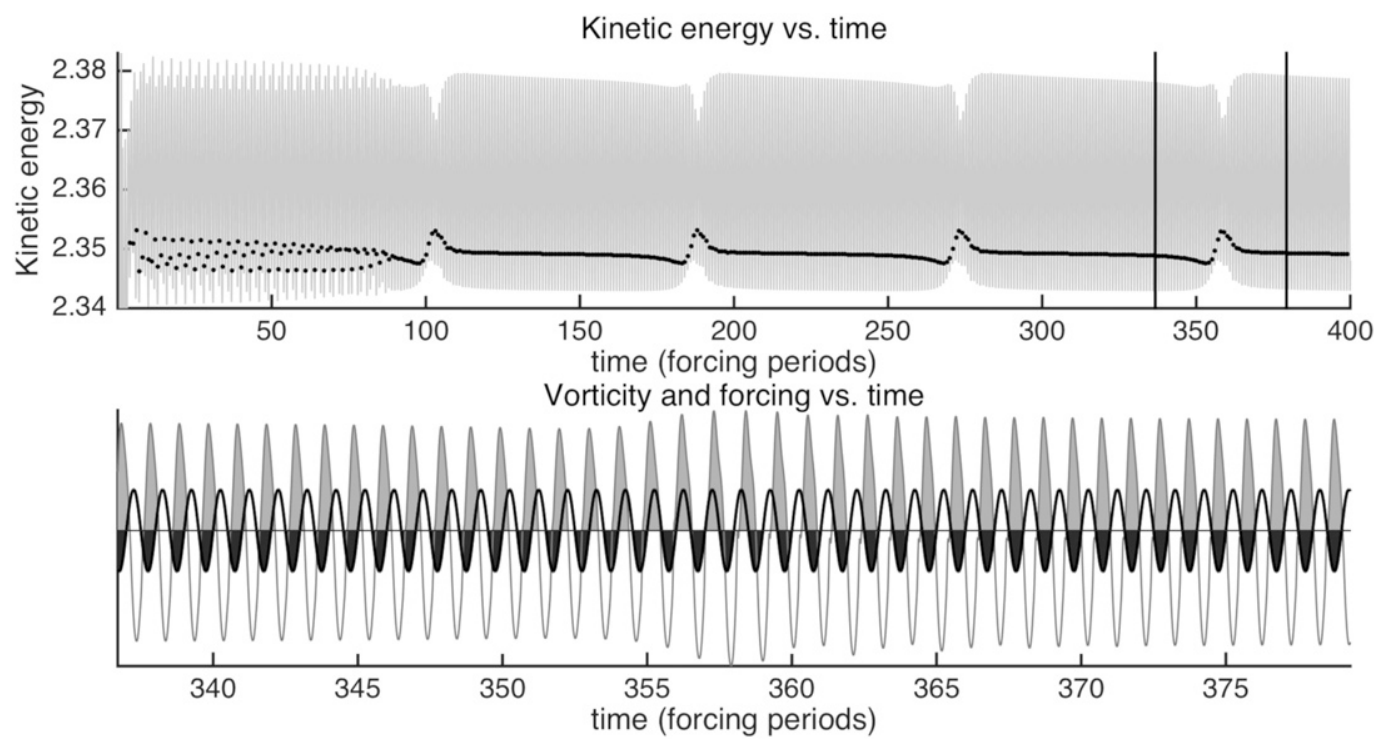

FIG. 8. Transition from an initial quasiperiodic state (almost $n: 6$ locked) to a final near-1:1 quasiperiodic state with phase slipping every 85.2 forcing cycles (continuing to the end of the run at $900 T_{w}$ ). (top) The $K(t)$ (gray) and $K(t)$ sampled every forcing period (dots). (bottom) Half a slipping cycle (between the lines in the top panel) showing forcing $W$ (black) and vorticity $\zeta$ in the eddy-shedding region (at radius 0.25 and azimuth $100^{\circ}$, gray), both scaled and offset vertically and with lower (upper) half of $W(\zeta)$ filled to facilitate phase comparison; Ro $=0.07\left(\delta_{I}=\right.$ $0.02124), A=5.714 \times 10^{-3}, T_{w} / T_{n}=0.95$.

by $f_{w}$. The eddy-shedding frequency $f_{s}=2 \pi / T_{s}$ also shifts away from $f_{n}=2 \pi / T_{n}$ toward $f_{w}$ for quasiperiodic states near a locked tongue. These phenomena are well known in other periodically forced nonlinear oscillators, and theory predicts $\left|f_{w}-f_{s}\right| \rightarrow 0$ at the tongue edge, that is, the time interval between slips increases without bound (Pikovsky et al. 2001). Figure 8 shows a case with $T_{w} / T_{n}=0.95$ where, after a quasiperiodic transient dominated by $1: 3$ and 1:6 harmonics, the system transitions permanently to another quasiperiodic state that is very close to being 1:1 locked. This final state has phase slips (in which the WBC loses a cycle relative to the forcing) every 85.2 forcing cycles, giving variance at time scales two orders of magnitude longer than the forcing or the intrinsic variability. Since $T_{w} / T_{n}=0.95$ we might expect slipping every $20 T_{w}$, but the slipping cycle is over four times slower because $T_{S}$ has shifted toward $T_{w}$. The amplitude of the $K$ cycle is reduced by about $30 \%$ during these brief slips away from nearsynchrony with the forcing.

In chaotic cases the $K$ spectrum is broad-banded and the delay space trajectories do not follow a predictable path. Cases of type 1 chaos were shown in Fig. 5. Figure 9 shows an example of type 2 chaos, in which the torus has disappeared and the intersections are no longer confined near a closed curve in the Poincaré section. This implies the presence of an additional intrinsic time scale, perhaps due to the excitation of otherwise-damped modes.
Chaos and phase slipping are particularly interesting because they introduce variability on long time scales not present in either the internal or external variability.

Under some conditions the time dependence changes abruptly from one type to another, for example, locked and chaotic states with long initial quasiperiodic transients, transitions to locked or quasiperiodic states following a chaotic transient, and transitions between different quasiperiodic states (Fig. 8). Locked states were unambiguously identified by exponential convergence of the diagnostic of Kiss (2007), but it is possible that a few of the states we labeled as chaotic or quasiperiodic may have actually been very long initial transients.

\section{e. Crossing thresholds}

When $A$ is sufficiently large, the forcing can transiently cross the thresholds $\mathrm{Ro}_{c 1,2,3,4}$, or even reverse sign (these thresholds are marked as horizontal lines in Fig. 4). If the period $T_{w}$ is very long compared to the gyre adjustment time scale (which is typically a few times $T_{n}$ ), we would expect the flow to progress through a range of the states identified in section $3 \mathrm{a}$ and to undergo bifurcations as these thresholds are crossed within each forcing cycle (Dewar 2003).

For $\mathrm{Ro}<\mathrm{Ro}_{c 1}$ the intrinsic variability is damped; however, if the variable forcing exceeds $\mathrm{Ro}_{c 1}$ at some time during its cycle the natural variability can potentially appear. Since the growth rate of the oscillatory 
(a)

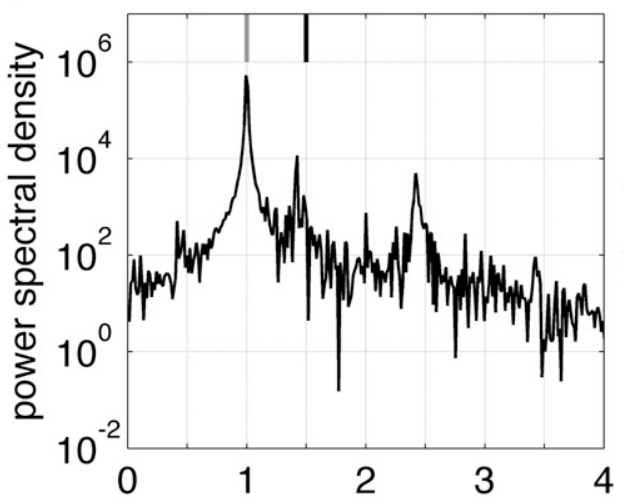

Frequency (cycles per forcing period) (b)

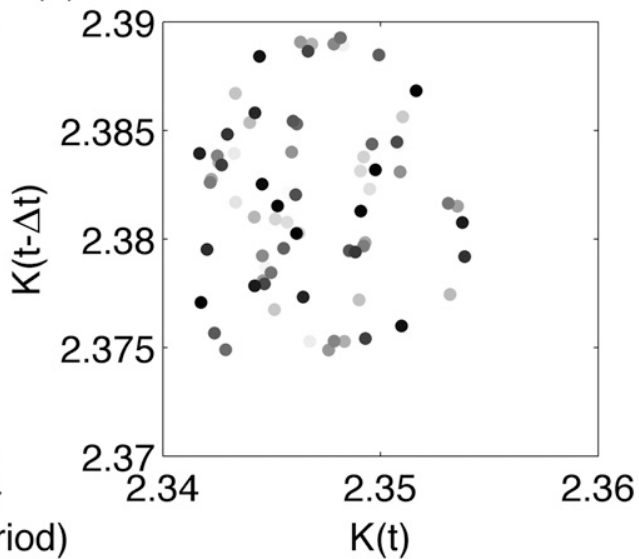

(c)

Kinetic energy anomaly vs. forcing phase

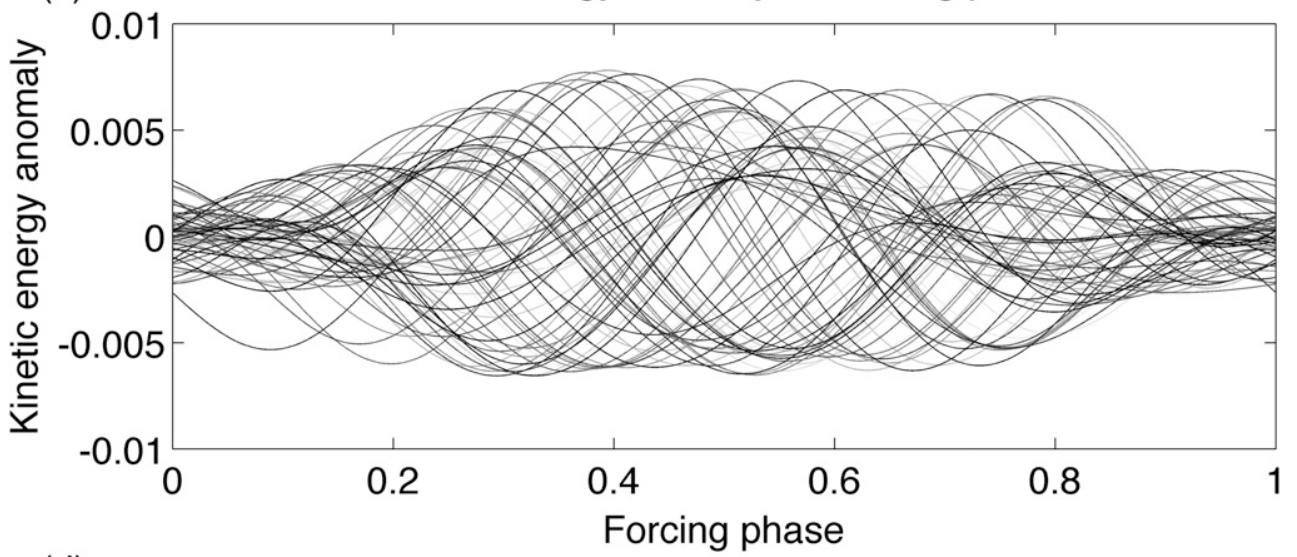

(d) Kinetic energy and forcing

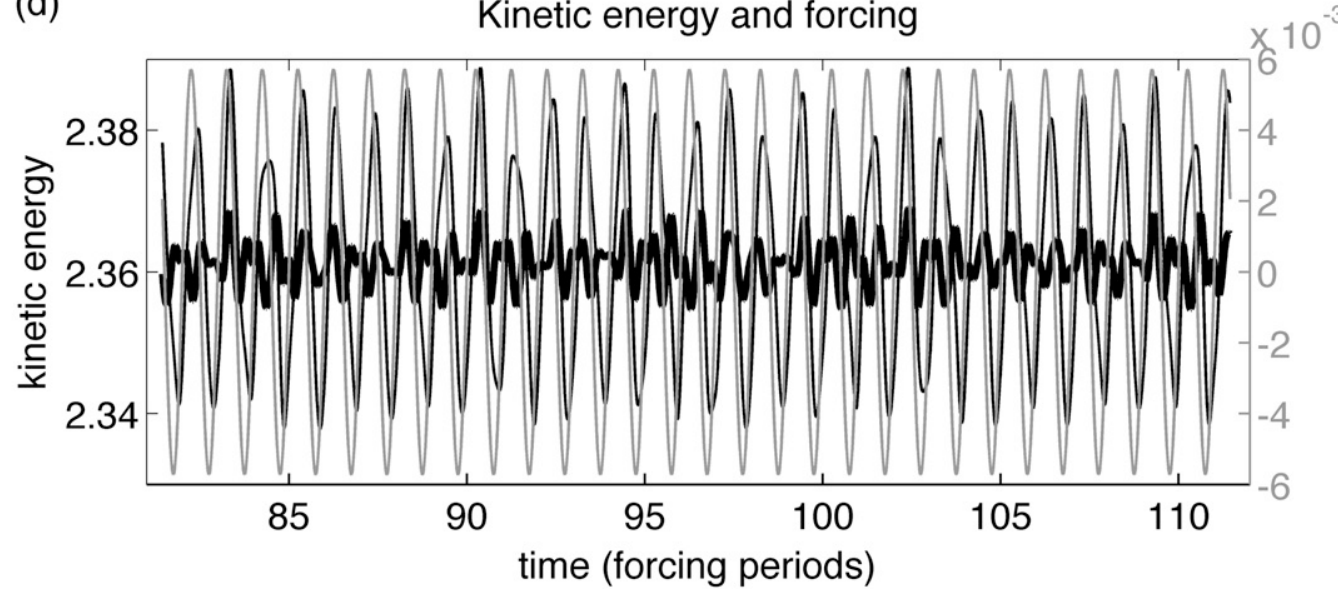

FIG. 9. As in Fig. 6, but for type 2 chaos for Ro $=0.07\left(\delta_{I}=0.02124\right), A=5.714 \times 10^{-3}, T_{w} / T_{n}=1.5$.

mode is small when $\mathrm{Ro} \approx \mathrm{Ro}_{c 1}$, large-amplitude, lowfrequency forcing is required so that there is sufficient time for the oscillations to develop before the forcing drops below the critical value. Figure 10 shows one such case, with $T_{w} / T_{n} \approx 12.9$ (using $T_{n}$ approximated from the decaying oscillations during spin up under steady forcing) and very large $A=0.85$, for Ro $=0.047$, just below $\mathrm{Ro}_{c 1}$. This forcing exceeds $\mathrm{Ro}_{c 1}$ for almost the entire positive half of the cycle. The time series of kinetic energy and vorticity show bursts of high-frequency variability indicative of eddy shedding that appear somewhat after the forcing exceeds the threshold and 


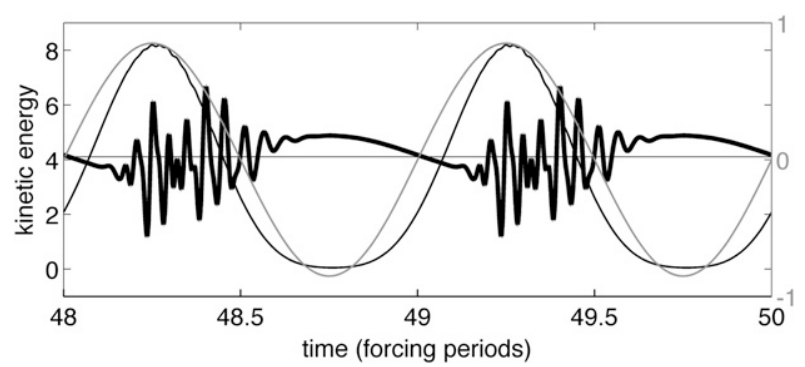

FIG. 10. Bursts of eddy shedding for Ro $=0.047\left(\delta_{I}=0.01740\right)$, $A=0.85, T_{w} / T_{n}=12.9$. As in Fig. $6 \mathrm{~d}$, but the thick black line is the vorticity $\zeta$ in the eddy-shedding region at radius 0.25 and azimuth $100^{\circ}$ (scaled and shifted vertically for comparison) and the horizontal line marks the forcing level corresponding to $\mathrm{Ro}_{c 1}$.

disappear somewhat after the forcing drops below the threshold. This delay reflects the time scale of growth and decay of the instability. Despite their complexity, these bursts of eddy shedding repeat exactly each forcing period in this example; thus, the whole cycle is phase locked.

\section{Discussion and conclusions}

Early conceptualizations of ocean variability considered the ocean to have no intrinsic modes of variability and to act as an integrator of its forcing (because of its long adjustment time scales), implying that red oceanic variability spectra arise in response to white stochastic forcing, and any narrow spectral peaks must originate from the forcing (e.g., Frankignoul and Hasselmann 1977). However, studies since the mid-1990s have shown how nonlinear processes can produce intrinsic variability under steady forcing (e.g., Dijkstra and Ghil 2005). Here we suggest that because of this nonlinear internal variability, the ocean may respond to variable forcing in much more complex ways than a simple integrator, in particular at frequencies that are absent from both the forcing and the steadily driven currents. We find several processes that can produce time scales $O(10-100)$ times longer than the natural or forcing periods in our model. This potential source of lowfrequency ocean variability has seldom been considered in studies of forced WBC variability because these typically use linear models that are incapable of responding in this way.

Under steady winds, our idealized gyre undergoes a Hopf bifurcation at a critical forcing strength, producing periodic eddy shedding at finite amplitude. Multiple states, period doubling, and chaos emerge through further bifurcations at stronger steady forcing, in agreement with the laboratory results of Griffiths and Kiss (1999). In the intrinsically periodic regime, temporally periodic wind forcing perturbs the internal variability, giving a response that depends on the strength of the mean forcing, the amplitude $A$ of the forcing variation, and especially the ratio of the forcing period $T_{w}$ to the intrinsic period $T_{n}$. The spatial structure of the variability is retained, but its temporal behavior can be significantly altered even with weak forcing variations of under $1 \%$.

The eddy shedding period can phase lock to a rational multiple of $T_{w}$ if $T_{w} / T_{n}$ is close to a simple rational; such locking can occur at greater mismatch at larger $A$, and has also been seen in laboratory experiments (Griffiths and Kiss 1999; Kiss 2007). If $T_{w}$ and $T_{n}$ are sufficiently incommensurate, then the eddy shedding may be quasiperiodic or, particularly for large mean forcing strengths, chaotic. The time scales of variability can be altered in all three cases. If phase locked, the eddyshedding frequency can be shifted significantly beyond its natural range to match a rational multiple of the forcing frequency, and multiple period doublings can also introduce low frequencies. If quasiperiodic, the eddy-shedding frequency can be shifted toward the forcing frequency (or a rational multiple), and very low frequencies can also arise from sums and differences of harmonics of the (shifted) natural and forcing frequencies, for example, in phase slipping. Chaotic states exhibit a broad range of time scales that extend to low frequencies. Chaos appears to arise via both the perioddoubling and quasiperiodic routes.

We find a complex dependence of the temporal response on the forcing frequency and amplitude. The location and ordering of phase-locked regions in many ways matches the "circle map" model of generic forced nonlinear oscillators, as does the dependence on initial conditions, but period-doubling cascades and chaos occur in unexpected parameter ranges. We attribute these differences to additional degrees of freedom, which are particularly evident in "type 2" chaotic states.

Of course we do not claim that a spatially uniform, temporally periodic wind stress curl is a realistic representation of the forcing of ocean gyres, or that results from a barotropic, flat-bottom, laminar model will translate directly to the real ocean. Our model is not intended to directly represent any particular gyre, but to serve as a simple test bed for the development of new concepts of ocean variability. Despite their lack of realism, previous idealized bifurcation studies have provided a valuable conceptual framework for interpretation of observed ocean variability, for example, in terms of the intrinsic "gyre mode" (Speich et al. 1995; Simonnet et al. 2003; Dijkstra and Ghil 2005). Similarly, a key objective of this paper is to provide examples of additional types of behavior that 
can arise under variable forcing and an organized framework in which they can be understood, with the hope that this will broaden the scope of mechanisms considered when interpreting ocean variability. We highlight new mechanisms by which long-time scale variability can arise and investigate the robustness of intrinsic modes to forcing variation. Berloff et al. (2007b) questioned the relevance of the gyre mode to a realistically turbulent ocean, and our results suggest that variable forcing could also disrupt the time dependence of such intrinsic modes. For example, an intrinsically periodic flow, far from the transition to chaos under steady forcing, can be made chaotic across a broad parameter range when the wind varies by as little as $1 \%$ (much less than the observed wind variation).

Circle-map-like dynamics are generic and occur in a diverse range of forced nonlinear oscillators in physical and biological systems, including those with aperiodic intrinsic and/or forcing variability (Pikovsky et al. 2001). Thus, these generic behaviors are to be expected a priori in nonlinear, intrinsically variable currents under variable forcing, irrespective of details such as the geometry of the current, the mechanism of intrinsic variability, or the form taken by the variable forcing. For example, Simonnet et al. (2003) and Sakamoto (2006) found evidence for synchronization between two intrinsic oscillatory modes in a two-gyre ocean model under steady wind forcing, and Chang et al. (1994), Jin et al. (1994, 1996), and Tziperman et al. (1994) argued that ENSO undergoes phase locking between an interannual coupled ocean-atmosphere mode and the seasonal cycle.

The relevance of our results to highly turbulent gyres far beyond the onset of chaos is an open question. While mesoscale baroclinic turbulence can play a fundamental role in some modes of low-frequency WBC variability (e.g., Berloff et al. 2007a,b; Dewar 2003; Kravtsov et al. 2006), the EAC, Agulhas, and Kuroshio have primary instabilities that seem to be largely barotropic (i.e., extracting energy from the horizontal shear) and captured in non-eddy-resolving models (Mata et al. 2006; Weijer et al. 2013; Pierini et al. 2009), suggesting that these instabilities are independent of (and robust to) baroclinic processes and turbulence. It therefore seems plausible that their response to variable forcing could be fruitfully studied in a laminar model lacking resolved baroclinic instability. Laminar dynamics are also relevant to understanding the variability of the non-eddy-resolving ocean components of present-generation (CMIP5/6) global coupled climate models.

In an eddy-resolving model the turbulent basic state under steady forcing has far higher dimension than a single oscillator. However, if the basic instability seen in laminar models is preserved, the turbulence may act on its dynamics like a noisy forcing (e.g., Berloff et al. 2007a). A realistic wind forcing would also include aperiodic and white-noise components in addition to an annual cycle. Phase locking cannot be exact in these situations, but may manifest as a nonuniform probability distribution with deep minima separating preferred phase differences between forcing and response (Pikovsky et al. 2001). Narrow high-order tongues would likely not survive noise from turbulence or forcing, but sufficiently weak noise may leave the centers of major tongues intact. We have conducted experiments (not shown) with white noise in addition to periodic forcing, and these show that the major locked tongues are indeed robust to moderate levels of noise, suggesting they could occur under more realistic forcing. We have also begun a study using a two-layer flat-bottomed gyre driven by the observed spatiotemporal annual wind cycle. This shows regimes of interleaved Arnold tongues phase locked to the forcing, despite having a more complex basic state and forcing, and disruption by baroclinic Rossby waves.

The amplitude of the annual WSC cycle is generally between 0.1 and 10 times the mean in the observational dataset of Risien and Chelton (2008). The WSC zonal phase variation and the phase lag due to barotropic Rossby wave propagation are small, so we might expect this to be equivalent to $0.1<A<10$ in our model, which is well into the range where we found strong synchronization effects (Fig. 4). However, bathymetry and stratification reduce the strength of the seasonal response (Myers and Weaver 1996; Sakamoto and Yamagata 1996), and bathymetry inhibits the propagation of barotropic Rossby waves that facilitate the barotropic response to variable forcing (Kindle 1991); thus, a topographic Sverdrup balance (governed by $\mathrm{f} / \mathrm{H}$ rather than $f$ ) is expected for the barotropic response to annual forcing (Anderson and Corry 1985). Also, our WBC is overly damped by bottom friction; we infer from Fig. 4 that under more realistically inertial conditions the primary effect of variable forcing would be to generate chaos rather than synchronization.

We investigated $T_{w} / T_{n}<2.3$, but the Agulhas and EAC shed major eddies roughly 4-6 and 3-4 times per year, respectively (Beal et al. 2011; Mata et al. 2006), giving $T_{w} / T_{n} \approx 3-6$ relative to the annual cycle, just outside our parameter range. A $T_{w}$ in the broad range of NAO and ENSO time scales is further outside our parameter range. However, circle-map theory predicts repeating behavior with increasing $T_{w} / T_{n}$ (Fig. 1), and in the two-layer model mentioned above we find similar locking structures near $T_{w} / T_{n}=1,2$, and 4 . Also, the annual WSC cycle has harmonics at 6,4 , and 3 months (Risien and Chelton 2008; Hong et al. 2000; DiNezio 
et al. 2009; Fu 2004), which are in the range we investigated relative to the natural time scales of these two currents. Interannual internal modes found under steady forcing also lie in our range relative to annual forcing [e.g., the gyre mode peaks at $T_{n} \approx 1.3,3.2$, and 6 years in Speich et al. (1995)].

Bathymetry, noisy forcing, strong inertia, and turbulence may explain the limited observational evidence for locking of WBC variability to the wind cycle. As discussed in section $1 \mathrm{~b}$, wind-forced variability in the Gulf Stream seems to contribute to its variability farther downstream. More strikingly, the strong annual WSC cycle in the northern Indian Ocean plays an important role (together with local wind forcing) in the seasonal reversal of the Somali Current and East Indian Coast Current. Annual-period baroclinic Rossby waves generated by coastal Kelvin waves on India's west coast and the WSC cycle in the western Arabian Sea combine with intrinsic instability of the Somali Current and local wind forcing to drive the annual formation of the Great Whirl against the Somali coast and eddies in the Gulf of Aden (Al Saafani et al. 2007; Bower and Furey 2012; Schott and McCreary 2001, and references therein). Annual cycles in the WBCs of the south Indian Ocean are much weaker (Schott and McCreary 2001), but they display strong variability at a period of around 90 days characteristic of Agulhas ring formation. The Agulhas seems to be intrinsically unstable, but the timing of ring shedding appears to be controlled by eddies arriving from upstream in the Mozambique Channel and East Madagascar Current, which in turn may be triggered by incoming Rossby waves (Schouten et al. 2002; Penven et al. 2006). These phenomena seem analogous to the triggering of eddy shedding by upstream perturbations in our model.

Acknowledgments. The research was funded, in part, by the ARC Centre of Excellence for Climate System Science (Grant CE110001028). We thank two anonymous reviewers for suggestions which have improved the manuscript.

\section{REFERENCES}

Al Saafani, M. A., S. S. C. Shenoi, D. Shankar, M. Aparna, J. Kurian, F. Durand, and P. N. Vinayachandran, 2007: Westward movement of eddies into the Gulf of Aden from the Arabian Sea. J. Geophys. Res., 112, C11004, doi:10.1029/2006JC004020.

Anderson, D. L. T., and A. E. Gill, 1975: Spin-up of a stratified ocean, with applications to upwelling. Deep-Sea Res. Oceanogr. Abstr., 22, 583-596, doi:10.1016/0011-7471(75)90046-7.

__ and R. A. Corry, 1985: Ocean response to low-frequency wind forcing with application to the seasonal-variation in the Florida Straits Gulf-Stream transport. Prog. Oceanogr., 14, 7-40, doi:10.1016/0079-6611(85)90003-5.

Aronson, D. G., R. P. McGehee, I. G. Kevrekidis, and R. Aris, 1986: Entrainment regions for periodically forced oscillators. Phys. Rev. A, 33, 2190-2192, doi:10.1103/PhysRevA.33.2190.
Baringer, M. O., and J. C. Larsen, 2001: Sixteen years of Florida Current transport at $27^{\circ}$ N. Geophys. Res. Lett., 28, 3179-3182, doi:10.1029/2001GL013246.

Beal, L. M., W. P. M. De Ruijter, A. Biastoch, and R. Zahn, 2011: On the role of the Agulhas system in ocean circulation and climate. Nature, 472, 429-436, doi:10.1038/nature09983.

Beardsley, R. C., 1969: A laboratory model of the wind-driven ocean circulation. J. Fluid Mech., 38, 255-271, doi:10.1017/ S0022112069000152.

_ 1975: The 'sliced-cylinder' laboratory model of the winddriven ocean circulation. Part 2: Oscillatory forcing and Rossby wave resonance. J. Fluid Mech., 69, 41-64, doi:10.1017/ S0022112075001309.

Becker, A., and M. A. Page, 1990: Flow separation and unsteadiness in a rotating sliced cylinder. Geophys. Astrophys. Fluid Dyn., 55, 89-115, doi:10.1080/03091929008203557.

Berloff, P. S., and J. C. McWilliams, 1999: Large-scale, lowfrequency variability in wind-driven ocean gyres. J. Phys. Oceanogr., 29, 1925-1949, doi:10.1175/1520-0485(1999)029<1925: LSLFVI $>2.0 . \mathrm{CO} ; 2$.

— W. Wewar, S. Kravtsov, and J. McWilliams, 2007a: Ocean eddy dynamics in a coupled ocean-atmosphere model. J. Phys. Oceanogr., 37, 1103-1121, doi:10.1175/JPO3041.1.

— A. M. Hogg, and W. K. Dewar, 2007b: The turbulent oscillator: A mechanism of low-frequency variability of the winddriven ocean gyres. J. Phys. Oceanogr., 37, 2363-2386, doi:10.1175/JPO3118.1.

Bowen, M. M., J. L. Wilkin, and W. J. Emery, 2005: Variability and forcing of the East Australian Current. J. Geophys. Res., 110, C03019, doi:10.1029/2004JC002533.

Bower, A. S., and H. H. Furey, 2012: Mesoscale eddies in the Gulf of Aden and their impact on the spreading of Red Sea outflow water. Prog. Oceanogr., 96, 14-39, doi:10.1016/ j.pocean.2011.09.003.

Chang, P., B. Wang, T. Li, and L. Ji, 1994: Interactions between the seasonal cycle and the southern oscillation-Frequency entrainment and chaos in a coupled ocean-atmosphere model. Geophys. Res. Lett., 21, 2817-2820, doi:10.1029/94GL02759.

Chave, A., D. Luther, and J. Filloux, 1991: Variability of the wind stress curl over the North Pacific: Implication for the oceanic response. J. Geophys. Res., 96, 18361-18379, doi:10.1029/91JC02152.

Chhak, K. C., A. M. Moore, and R. F. Milliff, 2009: Stochastic forcing of ocean variability by the North Atlantic Oscillation. J. Phys. Oceanogr., 39, 162-184, doi:10.1175/2008JPO3972.1.

Dewar, W. K., 2003: Nonlinear midlatitude ocean adjustment. J. Phys. Oceanogr., 33, 1057-1082, doi:10.1175/1520-0485(2003)033<1057: $\mathrm{NMOA}>2.0 . \mathrm{CO} ; 2$.

Dijkstra, H. A., 2000: Nonlinear Physical Oceanography: A Dynamical Systems Approach to the Large Scale Ocean Circulation and El Niño. Atmospheric and Oceanographic Sciences Library, Vol. 22, Kluwer Academic, 458 pp.

_ , and C. A. Katsman, 1997: Temporal variability of the winddriven quasi-geostrophic double gyre ocean circulation: Basic bifurcation diagrams. Geophys. Astrophys. Fluid Dyn., 85, 195-232, doi:10.1080/03091929708208989.

_ - and M. Ghil, 2005: Low-frequency variability of the largescale ocean circulation: A dynamical systems approach. Rev. Geophys., 43, RG3002, doi:10.1029/2002RG000122.

DiNezio, P. N., L. J. Gramer, W. E. Johns, C. S. Meinen, and M. O. Baringer, 2009: Observed interannual variability of the Florida Current: Wind forcing and the North Atlantic Oscillation. J. Phys. Oceanogr., 39, 721-736, doi:10.1175/ 2008JPO4001.1. 
Frankignoul, C., and K. Hasselmann, 1977: Stochastic climate models, Part II: Application to sea-surface temperature anomalies and thermocline variability. Tellus, 29A, 289-305, doi:10.1111/j.2153-3490.1977.tb00740.x.

_- N. Sennéchael, Y.-O. Kwon, and M. A. Alexander, 2011: Influence of the meridional shifts of the Kuroshio and the Oyashio extensions on the atmospheric circulation. J. Climate, 24, 762-777, doi:10.1175/2010JCLI3731.1.

Fu, L.-L., 2004: Latitudinal and frequency characteristics of the westward propagation of large-scale oceanic variability. J. Phys. Oceanogr., 34, 1907-1921, doi:10.1175/1520-0485(2004)034<1907: $\mathrm{LAFCOT}>2.0 . \mathrm{CO} ; 2$.

Glazier, J., and A. Libchaber, 1988: Quasi-periodicity and dynamical systems: An experimentalist's view. IEEE Trans. Circuits Syst., 35, 790-809, doi:10.1109/31.1826.

Griffiths, R. W., and A. E. Kiss, 1999: Flow regimes in a wide 'sliced-cylinder' model of homogeneous beta-plane circulation. J. Fluid Mech., 399, 205-236, doi:10.1017/ S0022112099006370.

Haucke, H., and R. Ecke, 1987: Mode-locking and chaos in RayleighBénard convection. Physica D, 25, 307-329, doi:10.1016/ 0167-2789(87)90106-0.

Hill, K. L., S. R. Rintoul, R. Coleman, and K. R. Ridgway, 2008: Wind forced low frequency variability of the East Australian Current. Geophys. Res. Lett., 35, L08602, doi:10.1029/2007GL032912.

Holbrook, N. J., I. D. Goodwin, S. McGregor, E. Molina, and S. B. Power, 2011: ENSO to multi-decadal time scale changes in East Australian Current transports and Fort Denison sea level: Oceanic Rossby waves as the connecting mechanism. Deep-Sea Res. II, 58, 547-558, doi:10.1016/j.dsr2.2010.06.007.

Hong, B. G., W. Sturges, and A. J. Clarke, 2000: Sea level on the U.S. east coast: Decadal variability caused by open ocean wind-curl forcing. J. Phys. Oceanogr., 30, 2088-2098, doi:10.1175/ 1520-0485(2000)030<2088:SLOTUS $>2.0 . \mathrm{CO} ; 2$.

$\mathrm{Hu}, \mathrm{D}$., and Coauthors, 2015: Pacific western boundary currents and their roles in climate. Nature, 522, 299-308, doi:10.1038/ nature14504.

Ierley, G. R., and V. A. Sheremet, 1995: Multiple solutions and advection-dominated flows in the wind-driven circulation. Part 1: Slip. J. Mar. Res., 53, 703-737, doi:10.1357/ 0022240953213052.

Jiang, S., F.-F. Jin, and M. Ghil, 1995: Multiple equilibria, periodic, and aperiodic solutions in a wind-driven, double-gyre, shallow-water model. J. Phys. Oceanogr., 25, 764-786, doi:10.1175/1520-0485(1995)025<0764:MEPAAS >2.0.CO;2.

Jin, F.-F., J. D. Neelin, and M. Ghil, 1994: El Niño on the Devil's Staircase: Annual subharmonic steps to chaos. Science, 264, 70-72, doi:10.1126/science.264.5155.70.

$\_, \ldots$, and —_ 1996: El Niño/Southern Oscillation and the annual cycle: Subharmonic frequency-locking and aperiodicity. Physica D, 98, 442-465, doi:10.1016/0167-2789(96)00111-X.

Johns, W. E., R. J. Zantopp, and G. Goni, 2003: Cross-gyre transport by North Brazil Current rings. Interhemispheric Water Exchange in the Atlantic Ocean, G. Goni and P. MalanotteRizzoli, Eds., Elsevier Oceanography Series, Vol. 68, Elsevier, 411-441, doi:10.1016/S0422-9894(03)80156-3.

Joyce, T., C. Deser, and M. Spall, 2000: The relation between decadal variability of subtropical mode water and the North Atlantic Oscillation. J. Climate, 13, 2550-2569, doi:10.1175/ 1520-0442(2000)013<2550:TRBDVO > 2.0.CO;2.

Kelly, K. A., R. J. Small, R. M. Samelson, B. Qiu, T. M. Joyce, Y.-O. Kwon, and M. F. Cronin, 2010: Western boundary currents and frontal air-sea interaction: Gulf Stream and
Kuroshio Extension. J. Climate, 23, 5644-5667, doi:10.1175/ 2010JCLI3346.1.

Kindle, J. C., 1991: Topographic effects on the seasonal circulation of the Indian Ocean. J. Geophys. Res., 96, 16827-16837, doi:10.1029/91JC01542.

Kiss, A. E., 2000: Dynamics of laboratory models of the wind-driven ocean circulation. Ph.D. thesis, Australian National University, 160 pp. [Available online at http://hdl.handle.net/1885/47497.]

_ 2002: Potential vorticity “crises", adverse pressure gradients, and western boundary current separation. J. Mar. Res., 60, 779-803, doi:10.1357/002224002321505138.

__ 2003: A modified quasigeostrophic formulation for weakly nonlinear barotropic flow with large-amplitude depth variations. Ocean Modell., 5, 171-191, doi:10.1016/ S1463-5003(02)00038-0.

- 2004: Potential vorticity dynamics in a domain with closed geostrophic contours. I: Steady linear flows. J. Mar. Res., 62, 461-489, doi:10.1357/0022240041850039.

_ 2007: Nonlinear resonance and chaos in an ocean model. Frontiers in Turbulence and Coherent Structures, J. Denier and J. S. Frederiksen, Eds., World Scientific Lecture Notes in Complex Systems, Vol. 6, World Scientific, 149-169.

- and C. Ménesguen, 2004: Response of ocean circulation to variable wind forcing. 15th Australasian Fluid Mechanics Conf., Sydney, NSW, Australia, University of Sydney, 4 pp. [Available online at http://sydney.edu.au/engineering/aeromech/ 15afmc/proceedings/papers/AFMC00145.pdf]

Kravtsov, S., P. Berloff, W. K. Dewar, M. Ghil, and J. C. McWilliams, 2006: Dynamical origin of low-frequency variability in a highly nonlinear midlatitude coupled model. J. Climate, 19, 6391-6408, doi:10.1175/JCLI3976.1.

Kwon, Y.-O., M. A. Alexander, N. A. Bond, C. Frankignoul, H. Nakamura, B. Qiu, and L. A. Thompson, 2010: Role of the Gulf Stream and Kuroshio-Oyashio systems in large-scale atmosphere-ocean interaction: A review. J. Climate, 23, 32493281, doi:10.1175/2010JCLI3343.1.

Large, W. G., W. R. Holland, and J. C. Evans, 1991: Quasi-geostrophic ocean response to real wind forcing: The effects of temporal smoothing. J. Phys. Oceanogr., 21, 998-1017, doi:10.1175/ 1520-0485(1991)021<0998:QGORTR > 2.0.CO;2.

Lee, T. N., W. E. Johns, R. J. Zantopp, and E. R. Fillenbaum, 1996: Moored observations of western boundary current variability and thermohaline circulation at $26.5^{\circ}$ in the subtropical North Atlantic. J. Phys. Oceanogr., 26, 962-983, doi:10.1175/ 1520-0485(1996)026<0962:MOOWBC >2.0.CO;2.

Marchesiello, P., and J. Middleton, 2000: Modeling the East Australian Current in the western Tasman Sea. J. Phys. Oceanogr., 30, 2956-2971, doi:10.1175/1520-0485(2001)031<2956: MTEACI $>2.0 . \mathrm{CO} ; 2$.

Mata, M. M., S. E. Wijffels, J. A. Church, and M. Tomczak, 2006: Eddy shedding and energy conversions in the East Australian Current. J. Geophys. Res., 111, C09034, doi:10.1029/2006JC003592.

McWilliams, J. C., 1977: A note on a consistent quasigeostrophic model in a multiply connected domain. Dyn. Atmos. Oceans, 1, 427-441, doi:10.1016/0377-0265(77)90002-1.

Molinari, R. L., 2004: Annual and decadal variability in the western subtropical North Atlantic: Signal characteristics and sampling methodologies. Prog. Oceanogr., 62, 33-66, doi:10.1016/ j.pocean.2004.07.002.

Myers, P., and A. Weaver, 1996: On the circulation of the North Pacific Ocean: Climatology, seasonal cycle and interpentadal variability. Prog. Oceanogr., 38, 1-49, doi:10.1016/ S0079-6611(96)00009-2. 
Nadiga, B. T., and B. P. Luce, 2001: Global bifurcation of Shilnikov type in a double-gyre ocean model. J. Phys. Oceanogr., 31, 2669-2690, doi:10.1175/1520-0485(2001)031<2669: GBOSTI $>2.0 . \mathrm{CO} ; 2$.

Nauw, J. J., and H. A. Dijkstra, 2001: The origin of low-frequency variability of double-gyre wind-driven flows. J. Mar. Res., 59, 567-597, doi:10.1357/002224001762842190.

Newhouse, S., D. Ruelle, and F. Takens, 1978: Occurrence of strange axiom $A$ attractors near quasi periodic flows on $T^{m}, m \geq 3$. Commun. Math. Phys., 64, 35-40, doi:10.1007/BF01940759.

Nilsson, C., and G. Cresswell, 1980: The formation and evolution of East Australian Current warm-core eddies. Prog. Oceanogr., 9, 133-183, doi:10.1016/0079-6611(80)90008-7.

Nof, D., 1996: Why are rings regularly shed in the western equatorial Atlantic but not in the western Pacific? Prog. Oceanogr., 38, 417-451, doi:10.1016/S0079-6611(97)00004-9.

Palastanga, V., P. J. van Leeuwen, and W. P. M. de Ruijter, 2006: A link between low-frequency mesoscale eddy variability around Madagascar and the large-scale Indian Ocean variability. J. Geophys. Res., 111, C09029, doi:10.1029/2005JC003081.

Pedlosky, J., and H. P. Greenspan, 1967: A simple laboratory model for the oceanic circulation. J. Fluid Mech., 27, 291-304, doi:10.1017/S0022112067000321.

Penven, P., J. R. E. Lutjeharms, and P. Florenchie, 2006: Madagascar: A pacemaker for the Agulhas Current system? Geophys. Res. Lett., 33, L17609, doi:10.1029/2006GL026854.

Pierini, S., 2010: Coherence resonance in a double-gyre model of the Kuroshio Extension. J. Phys. Oceanogr., 40, 238-248, doi:10.1175/2009JPO4229.1.

-, 2011: Low-frequency variability, coherence resonance, and phase selection in a low-order model of the wind-driven ocean circulation. J. Phys. Oceanogr., 41, 1585-1604, doi:10.1175/ JPO-D-10-05018.1.

— H. A. Dijkstra, and A. Riccio, 2009: A nonlinear theory of the Kuroshio Extension bimodality. J. Phys. Oceanogr., 39, 2212-2229, doi:10.1175/2009JPO4181.1.

Pikovsky, A., M. Rosenblum, and J. Kurths, 2001: Synchronization: A Universal Concept in Nonlinear Sciences. Cambridge Nonlinear Science Series, Vol. 12, Cambridge University Press, 432 pp.

Qiu, B., 2003: Kuroshio Extension variability and forcing of the Pacific decadal oscillations: Responses and potential feedback. J. Phys. Oceanogr., 33, 2465-2482, doi:10.1175/2459.1. , and S. Chen, 2004: Seasonal modulations in the eddy field of the South Pacific Ocean. J. Phys. Oceanogr., 34, 1515-1527, doi:10.1175/1520-0485(2004)034<1515:SMITEF>2.0.CO;2.

— , and — 2005: Variability of the Kuroshio Extension jet, recirculation gyre, and mesoscale eddies on decadal time scales. J. Phys. Oceanogr., 35, 2090-2103, doi:10.1175/JPO2807.1.

Ridgway, K. R., and J. S. Godfrey, 1997: Seasonal cycle of the East Australian Current. J. Geophys. Res., 102, 22 921-22 936, doi:10.1029/97JC00227.

Risien, C. M., and D. B. Chelton, 2008: A global climatology of surface wind and wind stress fields from eight years of QuikSCAT scatterometer data. J. Phys. Oceanogr., 38, 2379-2413, doi:10.1175/2008JPO3881.1.

Rosenblum, M., and A. Pikovsky, 2003: Synchronization: From pendulum clocks to chaotic lasers and chemical oscillators. Contemp. Phys., 44, 401-416, doi:10.1080/ 00107510310001603129.

Sakamoto, T., 2006: Low-frequency variability of a two-layer ocean forced by periodic winds. Earth Planets Space, 58, 1203-1212, doi:10.1186/BF03352011.
__ and T. Yamagata, 1996: Seasonal transport variations of the wind-driven ocean circulation in a two-layer planetary geostrophic model with a continental slope. J. Mar. Res., 54, 261-284, doi:10.1357/0022240963213402.

Sapsis, T. P., and H. A. Dijkstra, 2013: Interaction of additive noise and nonlinear dynamics in the double-gyre wind-driven ocean circulation. J. Phys. Oceanogr., 43, 366-381, doi:10.1175/ JPO-D-12-047.1.

Schmeits, M., and H. Dijkstra, 2001: Bimodal behavior of the Kuroshio and the Gulf Stream. J. Phys. Oceanogr., 31, 3435-3456, doi:10.1175/1520-0485(2001)031<3435:BBOTKA >2.0.CO;2.

Schott, F. A., and J. P. McCreary Jr., 2001: The monsoon circulation of the Indian Ocean. Prog. Oceanogr., 51, 1-123, doi:10.1016/S0079-6611(01)00083-0.

Schouten, M. W., W. P. M. de Ruijter, and P. J. van Leeuwen, 2002: Upstream control of Agulhas Ring shedding. J. Geophys. Res., 107, 3109, doi:10.1029/2001JC000804

Shimokawa, S., and T. Matsuura, 2010: Chaotic behaviors in the response of a quasigeostrophic oceanic double gyre to seasonal external forcing. J. Phys. Oceanogr., 40, 1458-1472, doi:10.1175/2010JPO4400.1.

Simonnet, E., M. Ghil, K. Ide, R. Temam, and S. H. Wang, 2003: Low-frequency variability in shallow-water models of the wind-driven ocean circulation. Part II: Time-dependent solutions. J. Phys. Oceanogr., 33, 729-752, doi:10.1175/ 1520-0485(2003)33<729:LVISMO > 2.0.CO;2.

Sloyan, B. M., and T. J. O'Kane, 2015: Drivers of decadal variability in the Tasman Sea. J. Geophys. Res. Oceans, 120, 3193-3210, doi:10.1002/2014JC010550.

Speich, S., H. A. Dijkstra, and M. Ghil, 1995: Successive bifurcations in a shallow-water model applied to the wind-driven ocean circulation. Nonlinear Processes Geophys., 2, 241-268, doi:10.5194/npg-2-241-1995.

Sura, P., K. Fraedrich, and F. Lunkeit, 2001: Regime transitions in a stochastically forced double-gyre model. J. Phys. Oceanogr., 31, 411-426, doi:10.1175/1520-0485(2001)031<0411: RTIASF $>2.0 . \mathrm{CO} ; 2$.

Sushama, L., M. Ghil, and K. Ide, 2007: Spatio-temporal variability in a mid-latitude ocean basin subject to periodic wind forcing. Atmos.-Ocean, 45, 227-250, doi:10.3137/ao.450404.

Trenberth, K. E., J. G. Olson, and W. G. Large, 1989: A global ocean wind stress climatology based on ECMWF analyses. NCAR Tech. Note NCAR/TN-338+STR, 93 pp., doi:10.5065/ D6ST7MR9.

_ W. G. Large, and J. G. Olson, 1990: The mean annual cycle in global ocean wind stress. J. Phys. Oceanogr., 20, 1742-1760, doi:10.1175/1520-0485(1990)020<1742:TMACIG >2.0.CO;2.

Tziperman, E., L. Stone, M. A. Cane, and H. Jarosh, 1994: El Niño chaos: Overlapping of resonances between the seasonal cycle and the Pacific ocean-atmosphere oscillator. Science, 264, 72-74, doi:10.1126/science.264.5155.72.

van der Vaart, P. C. F., H. M. Schuttelaars, D. Calvete, and H. A. Dijkstra, 2002: Instability of time-dependent wind-driven ocean gyres. Phys. Fluids, 14, 3601-3615, doi:10.1063/1.1503804.

Weijer, W., V. Zharkov, D. Nof, H. A. Dijkstra, W. P. M. de Ruijter, A. T. van Scheltinga, and F. Wubs, 2013: Agulhas ring formation as a barotropic instability of the retroflection. Geophys. Res. Lett., 40, 5435-5438, doi:10.1002/2013GL057751.

Willebrand, J., S. G. H. Philander, and R. C. Pacanowski, 1980: The oceanic response to large-scale atmospheric disturbances. J. Phys. Oceanogr., 10, 411-429, doi:10.1175/1520-0485(1980)010<0411: TORTLS $>2.0 . \mathrm{CO} ; 2$. 